\title{
THE WRIGHT DECODER: A PAGE INDEX TO THE CATALOGUE OF SYRIAC MANUSCRIPTS IN THE BRITISH MUSEUM
}

\author{
ELIZABETH REIF \\ PRINCETON THEOLOGICAL SEMINARY \\ MiCHAEL PENN \\ MOUNT HOLYOKE COLLEGE
}

\begin{abstract}
The British Library contains the world's largest collection of extant Syriac manuscripts. Unfortunately, its principal catalog, William Wright's Catalogue of Syriac Manuscripts in the British Museum Acquired since the Year 1838, does not include a page index. This has resulted in great frustration and loss of research time for those who have consulted the British Library holdings. The "Wright Decoder" provides the index we all wish William Wright had himself written. It is organized by sequential shelfmarks and provides the volume and page numbers in Wright's catalog that corresponds to each Syriac manuscript.
\end{abstract}

With a collection of over a thousand Syriac manuscripts including the majority of those written in the first millennium, the British Library is the world's most important depository of original Syriac material. In the 1870s William Wright catalogued most of the 
British Library's Syriac holdings and his three volume Catalogue of Syriac Manuscripts in the British Museum Acquired since the Year 1838 remains an indispensible resource for all researchers of Syriac studies. ${ }^{1}$ Wright carefully inventoried the content of the collection, provided basic codicological information for each manuscript, estimated the date of composition, and often pointed out juicy tidbits about the scribes who wrote these manuscripts-ranging from their often elaborately composed anathemas to complaints against biting flies, bad parchment, and no one reading the manuscripts they so laboriously had copied. Despite its numerous strengths and riches there remains, however, one lacuna in Wright's Catalogue that has irked generations of Syriac scholars. It does not have a page index.

Unlike his later catalog of the Cambridge University Library, ${ }^{2}$ Wright's catalog of the British Museum's (later British Library's) collection does not proceed in order of shelfmark. Instead, he organized his Catalogue by genre (e.g. biblical manuscripts, hagiographies, scientific texts, etc.). As a result, the progression of the Catalogue's 1200 pages has no relationship with shelfmark. Adjacent shelfmarks may be separated by a thousand pages while manuscripts found sequentially in Wright's catalogue may have shelfmarks that differ by five thousand. To help the reader navigate through his Catalogue Wright included in the final volume a list of all the shelfmarks. Unfortunately, this master index does not provide page numbers, most likely because when Wright composed it he did not have the equivalent of modern day page proofs.

Instead Wright assigned a Roman numeral to each manuscript depending on where it appeared in his catalogue and he had his final index correlate this numeral with the shelf mark. So, for example, the first manuscript appearing in Wright's Catalogue (Egerton 704) was assigned the notation of "I," the second (Additional 14,671) "II" and so forth. These numerals, however, have no further descriptive function; one neither cites nor orders a

${ }^{1}$ William Wright, Catalogue of Syriac Manuscripts in the British Museum Acquired since the Year 1838. 3 Volumes (London: Cambridge University Press, 1870-1873). Reprinted by Gorgias Press in 2002.

${ }^{2}$ William Wright, A catalogue of the Syriac manuscripts preserved in the Library of the University of Cambridge. 2 Volumes (Cambridge: Cambridge University Press, 1901). Reprinted by Gorgias Press in 2002. 
Syriac manuscript using them. So what do you do if you want to look at manuscript Additional 17,178, for example? First you go to Wright's index and read that Additional 17,178 corresponds to DCCCXXVIII. But now what? There is no indication as to what volume or page this corresponds to in the Catalogue. If you are lucky enough to guess that this appears in the second volume, you then start flipping through the pages to zero in on where DCCCXXVIII lies. Alas, Wright's numbers do not appear on each page, but only as a header to a given manuscript. So, you may have to flip through a dozen pages to move from one Roman numeral to another. Alternatively, a given page might contain three or four numerals. Worse yet, what if your ability to memorize Roman numerals is a little off and you forget one of the C's. Only ten minutes later when you finally make it to the end of page 639 will you read in small print the actual shelfmark and discover your mistake (DCCXXVIII is Additional 14,610 not 17,178). You then have to go through over two hundred more pages before you end up on page 855 which is the proper place to find Additional 17,1798 (aka. DCCCXXVIII). Although simply inconvenient when looking for a single manuscript, this becomes quite frustrating when looking for multiple manuscripts.

The "Wright Decoder" is the index we all wish William Wright had himself written. Although not the sort of article you would wish to read from start to finish, this master index can save scholars countless hours if simply printed out and enclosed with one's own copy of Wright's Catalogue or if brought directly to the British Library. It is organized by sequential shelfmarks that immediately provide the Catalogue volume and page numbers corresponding to that manuscript. It also includes Wright's own numeration as well as his paleographic assessment of the manuscript's date. The hope is that this may be a time saving device for those scholars who consult Wright's Catalogue as well as those who have pleasure of working directly with the British Library collection. 


\begin{tabular}{|c|c|c|c|}
\hline Shelfmark & $\begin{array}{l}\text { Catalog pp. } \\
\text { (volume. } \\
\text { pages) }\end{array}$ & $\begin{array}{l}\text { Wright's } \\
\text { Numeration }\end{array}$ & Wright's Dating \\
\hline $\begin{array}{l}\text { Add. 12,133, } \\
\text { foll. 1-108 }\end{array}$ & $1.7-8$ & IX & Approx. 8c (early) \\
\hline $\begin{array}{l}\text { Add. 12,133, } \\
\text { foll. 109-169 }\end{array}$ & $1.31-32$ & LI & Approx. 8c \\
\hline Add. 12,134 & $1.29-31$ & XLIX & 697 \\
\hline $\begin{array}{l}\text { Add. 12,135, } \\
\text { foll. 1-43 }\end{array}$ & $1.24-26$ & XL & 726 \\
\hline $\begin{array}{l}\text { Add. 12,135, } \\
\text { foll. 44-207 }\end{array}$ & $2.486-487$ & DCXIII & 611 \\
\hline Add. 12,136 & 1.24 & XXXIX & Approx. 7c \\
\hline Add. 12,137 & $1.50-52$ & LXXV & Approx. 6c-7c \\
\hline Add. 12,138 & $1.101-108$ & CLXI & 899 \\
\hline Add. 12,139 & $1.154-159$ & CCXXIV & 1000 \\
\hline Add. 12,140 & 1.49 & LXXIII & Approx. 6c \\
\hline Add. 12,141 & $1.63-64$ & LXXXIX & Approx. 6c-7c \\
\hline $\begin{array}{l}\text { Add. 12,142, } \\
\text { foll. 1-73 }\end{array}$ & $1.97-98$ & CLIV & Approx. 6c-7c \\
\hline $\begin{array}{l}\text { Add. 12,142, } \\
\text { foll. 108-242 }\end{array}$ & $2.465-466$ & DLXXXII & Approx. 6c (early) \\
\hline $\begin{array}{l}\text { Add. 12,142, } \\
\text { foll. } 74-107\end{array}$ & $3.1092-1093$ & DCCCCXLIV & Approx. 6c \\
\hline Add. 12,143 & 2.623 & DCCXXII & 1229 \\
\hline Add. 12,144 & $2.908-914$ & DCCCLIII & 1081 \\
\hline Add. 12,145 & $1.251-254$ & CCCXIII & Approx. 10c \\
\hline Add. 12,146 & $1.258-261$ & CCCXIX & 1007 \\
\hline Add. 12,147 & $1.261-264$ & CCCXX & 1006 \\
\hline Add. 12,148 & $1.264-266$ & CCCXXI & 1007 \\
\hline Add. 12,149 & $1.266-269$ & CCCXXII & 1006 \\
\hline Add. 12,150 & $2.631-633$ & DCCXXVI & 412 \\
\hline
\end{tabular}




\begin{tabular}{|l|l|l|l|}
\hline Shelfmark & $\begin{array}{l}\text { Catalog pp. } \\
\text { (volume. } \\
\text { pages) }\end{array}$ & $\begin{array}{l}\text { Wright's } \\
\text { Numeration }\end{array}$ & Wright's Dating \\
\hline Add. 12,151 & $2.493-497$ & DCXXV & 804 \\
\hline Add. 12,152 & $2.497-499$ & DCXXVI & 837 \\
\hline Add. 12,153 & $2.423-428$ & DLV & 845 \\
\hline Add. 12,154 & $2.976-989$ & DCCCLX & $\begin{array}{l}\text { Approx. 8c (late)- } \\
\text { 9c (early) }\end{array}$ \\
\hline Add. 12,155 & $2.921-955$ & DCCCLVII & Approx. 8c \\
\hline Add. 12,156 & $2.639-648$ & DCCXXIX & Approx. 6c (<562) \\
\hline Add. 12,157 & $2.550-554$ & DCLXXXVIII & Approx. 7c-8c \\
\hline Add. 12,158 & $2.555-557$ & DCXC & Approx. 6c (588) \\
\hline Add. 12,159 & $2.534-546$ & DCLXXXV & 868 \\
\hline $\begin{array}{l}\text { Add. 12,160, } \\
\text { foll. 1-108 }\end{array}$ & $2.472-473$ & DXC & 584 \\
\hline $\begin{array}{l}\text { Add. 12,160, } \\
\text { foll. 109-185 }\end{array}$ & $3.1090-1091$ & DCCCCXLII & Approx. 6c (late) \\
\hline Add. 12,161 & $2.469-470$ & DLXXXVII & Approx. 6c-7c \\
\hline Add. 12,162 & $2.721-723$ & DCCLIX & Approx. 9c \\
\hline $\begin{array}{l}\text { Add. 12,163, } \\
\text { foll. 1-126 }\end{array}$ & $2.529-530$ & DCLXXVII & Approx. 6c \\
\hline $\begin{array}{l}\text { Add. 12,163, } \\
\text { foll. 127-304 }\end{array}$ & $2.834-836$ & DCCCXIX & Approx. 10c-11c \\
\hline $\begin{array}{l}\text { Add. 12,163, } \\
\text { foll. 305-311 }\end{array}$ & 2.445 & DLXVI & Approx. 7c \\
\hline Add. 12,164 & $2.527-529$ & DCLXXVI & Approx. 6c \\
\hline Add. 12,165 & $2.842-851$ & DCCCXXV & 1015 \\
\hline $\begin{array}{l}\text { Add. 12,166, } \\
\text { foll. 1-154 }\end{array}$ & $2.674-676$ & DCCXLII & Approx. 6c \\
\hline $\begin{array}{l}\text { Add. 12,166, } \\
\text { foll. 155-258 }\end{array}$ & $2.491-492$ & DCXX & 553 \\
\hline Add. 12,167 & $2.769-774$ & DCCLXXXV & 876 \\
\hline Add. 12,168 & $2.904-908$ & DCCCLII & Approx. 8c-9c \\
\hline
\end{tabular}




\begin{tabular}{|c|c|c|c|}
\hline Shelfmark & $\begin{array}{l}\text { Catalog pp. } \\
\text { (volume. } \\
\text { pages) }\end{array}$ & $\begin{array}{l}\text { Wright's } \\
\text { Numeration }\end{array}$ & Wright's Dating \\
\hline $\begin{array}{l}\text { Add. 12,169, } \\
\text { foll. 1-178 }\end{array}$ & $2.589-590$ & DCCIII & Approx. 8c-9c \\
\hline $\begin{array}{l}\text { Add. 12,169, } \\
\text { foll. 179-218 }\end{array}$ & $2.673-674$ & DCCXLI & Approx. 6c \\
\hline $\begin{array}{l}\text { Add. 12,170, } \\
\text { foll. 1-135 }\end{array}$ & $2.458-461$ & DLXXV & 604 \\
\hline $\begin{array}{l}\text { Add. 12,170, } \\
\text { foll. 136-276 }\end{array}$ & $2.746-752$ & DCCLXXIV & $\begin{array}{l}\text { Approx. } \quad 8 c-9 c \\
\text { (early) }\end{array}$ \\
\hline $\begin{array}{l}\text { Add. 12,170, } \\
\text { foll. } 277-279\end{array}$ & 2.752 & DCCLXXV & $\begin{array}{l}\text { Approx. } \quad 8 c-9 c \\
\text { (early) }\end{array}$ \\
\hline $\begin{array}{l}\text { Add. 12,171, } \\
\text { foll. 1-64 }\end{array}$ & $2.587-588$ & DCCI & 815 \\
\hline $\begin{array}{l}\text { Add. 12,171, } \\
\text { foll. } 65-68\end{array}$ & $2.766-767$ & DCCLXXXII & 833 \\
\hline $\begin{array}{l}\text { Add. 12,172, } \\
\text { foll. 1-11 }\end{array}$ & 2.465 & DLXXX & Approx. 10c \\
\hline $\begin{array}{l}\text { Add. 12,172, } \\
\text { foll. 12-24 }\end{array}$ & $3.1116-1117$ & DCCCCLIII & Approx. 10c \\
\hline $\begin{array}{l}\text { Add. 12,172, } \\
\text { foll. 136-196 }\end{array}$ & $1.6-7$ & VII & $\begin{array}{l}\text { Approx. 9c-10c, } \\
10 c-11 c\end{array}$ \\
\hline $\begin{array}{l}\text { Add. 12,172, } \\
\text { foll. 197-234 }\end{array}$ & $1.12-13$ & XVIII & Approx. 6c-7c \\
\hline $\begin{array}{l}\text { Add. 12,172, } \\
\text { foll. 25-54 }\end{array}$ & $3.1117-1118$ & DCCCCLIV & Approx. 10c \\
\hline $\begin{array}{l}\text { Add. 12,172, } \\
\text { foll. } 55-64\end{array}$ & 2.608 & DCCXII & Approx. 9c \\
\hline $\begin{array}{l}\text { Add. 12,172, } \\
\text { foll. } 65-135\end{array}$ & $2.592-605$ & DCCVII & Approx. 9c \\
\hline Add. 12,173 & $3.1070-1072$ & DCCCCXXIII & Approx. 6c-7c \\
\hline Add. 12,174 & $\begin{array}{l}3.1123- \\
3.1139\end{array}$ & DCCCCLX & 1197 \\
\hline $\begin{array}{l}\text { Add. 12,175, } \\
\text { foll. 1-48 }\end{array}$ & $3.1100-1101$ & DCCCCXLVI & Approx. 7c-8c \\
\hline
\end{tabular}




\begin{tabular}{|c|c|c|c|}
\hline Shelfmark & $\begin{array}{l}\text { Catalog pp. } \\
\text { (volume. } \\
\text { pages) }\end{array}$ & $\begin{array}{l}\text { Wright's } \\
\text { Numeration }\end{array}$ & Wright's Dating \\
\hline $\begin{array}{l}\text { Add. 12,175, } \\
\text { foll. 49-80 }\end{array}$ & $2.657-658$ & DCCXXXVI & Approx. 6c \\
\hline $\begin{array}{l}\text { Add. 12,175, } \\
\text { foll. } 81-254\end{array}$ & $2.633-638$ & DCCXXVII & 534 \\
\hline Add. 12,176 & $2.409-410$ & DXXXVII & Approx. 5c-6c \\
\hline Add. 12,177 & $1.58-60$ & LXXXIII & 1189 \\
\hline Add. 12,178 & $1.108-111$ & CLXII & Approx. 9c-10c \\
\hline Add. 12,179 & $1.321-324$ & CCCCX & Approx. 11c-12c \\
\hline Add. 12,180 & $2.474-475$ & DXCII & $\begin{array}{ll}\text { Approx. } & \text { 6c- } \\
\text { Approx. 7c }\end{array}$ \\
\hline Add. 12,181 & $2.558-564$ & DCXCII & Approx. 8c \\
\hline Add. 14, 562 & $2.470-471$ & DLXXXVIII & Approx. 7c-8c \\
\hline $\begin{array}{l}\text { Add. 14, 669, } \\
\text { foll. } 27,28\end{array}$ & 1.69 & CIV & Approx. 7c \\
\hline $\begin{array}{l}\text { Add. 14, 738, } \\
\text { foll. 79-81 }\end{array}$ & 1.377 & CCCCLXXVII & Approx. 13c \\
\hline Add. 14,425 & $1.3-5$ & III & 464 \\
\hline Add. 14,426 & 1.6 & $\mathrm{~V}$ & Approx. 6c-7c \\
\hline Add. 14,427 & $1.5-6$ & IV & Approx. 6c \\
\hline Add. 14,428 & $1.9-10$ & $\mathrm{XI}$ & 724 \\
\hline Add. 14,429 & $1.37-39$ & LX & 719 \\
\hline Add. 14,430 & $1.15-16$ & XXIV & 724 \\
\hline Add. 14,431 & $1.14-15$ & XXII & 545 \\
\hline Add. 14,432 & 1.21 & XXXIV & Approx. 6c \\
\hline Add. 14,433 & 1.126 & CLXXVII & Approx. 10c \\
\hline $\begin{array}{l}\text { Add. 14,434, } \\
\text { foll. 1-79 }\end{array}$ & $1.35-36$ & LIV & Approx. 8c (early) \\
\hline $\begin{array}{l}\text { Add. 14,434, } \\
\text { foll. } 80-128\end{array}$ & $1.36-37$ & $\mathrm{LV}$ & Approx. 8c \\
\hline
\end{tabular}




\begin{tabular}{|c|c|c|c|}
\hline Shelfmark & $\begin{array}{l}\text { Catalog pp. } \\
\text { (volume. } \\
\text { pages) }\end{array}$ & $\begin{array}{l}\text { Wright's } \\
\text { Numeration }\end{array}$ & Wright's Dating \\
\hline Add. 14,435 & $1.122-123$ & CLXXI & Approx. 9c \\
\hline $\begin{array}{l}\text { Add. } 14,436 \text {, } \\
\text { foll. } 1-76\end{array}$ & $1.119-120$ & CLXIX & Approx. 8c-9c \\
\hline $\begin{array}{l}\text { Add. 14,436, } \\
\text { foll. } 77-129\end{array}$ & $1.126-127$ & CLXXVIII & Approx. 10c \\
\hline $\begin{array}{l}\text { Add. 14,437, } \\
\text { foll. 1-46 }\end{array}$ & 1.31 & $\mathrm{~L}$ & Approx. 8c \\
\hline $\begin{array}{l}\text { Add. 14,437, } \\
\text { foll. } 47-124\end{array}$ & $1.33-34$ & LIII & Approx. 8c \\
\hline $\begin{array}{l}\text { Add. 14,438, } \\
\text { foll. 1-49 }\end{array}$ & 1.10 & XII & Approx. 6c \\
\hline $\begin{array}{l}\text { Add. 14,438, } \\
\text { foll. } 50-101\end{array}$ & $1.13-14$ & XXI & Approx. 6c \\
\hline Add. 14,439 & 1.13 & XIX & Approx. 6c-7c \\
\hline Add. 14,440 & $1.10-11$ & XVI & Approx. 10c-11c \\
\hline Add. 14,441 & 1.39 & LXI & 719 \\
\hline $\begin{array}{l}\text { Add. 14,442, } \\
\text { foll. 1-46 }\end{array}$ & $1.28-29$ & XLVIII & Approx. 7c \\
\hline $\begin{array}{l}\text { Add. 14,442, } \\
\text { foll. 47-65 }\end{array}$ & 1.15 & XXIII & Approx. 6c-7c \\
\hline $\begin{array}{l}\text { Add. 14,443, } \\
\text { foll. 1-34 }\end{array}$ & 1.17 & XXVI & Approx. 6c \\
\hline $\begin{array}{l}\text { Add. 14,443, } \\
\text { foll. } 35-71\end{array}$ & 1.18 & XXX & Approx. 6c-7c \\
\hline $\begin{array}{l}\text { Add. 14,443, } \\
\text { foll. } 72-98\end{array}$ & 1.18 & XXXI & Approx. 6c-7c \\
\hline $\begin{array}{l}\text { Add. 14,443, } \\
\text { foll. 99-144 }\end{array}$ & 1.27 & XLII & Approx. 6c \\
\hline $\begin{array}{l}\text { Add. 14,444, } \\
\text { foll. 1-24 }\end{array}$ & 1.6 & VI & Approx. 6c-7c \\
\hline $\begin{array}{l}\text { Add. 14,444, } \\
\text { foll. } 25-38\end{array}$ & 1.8 & $\mathrm{X}$ & Approx. 6c \\
\hline
\end{tabular}




\begin{tabular}{|l|l|l|l|}
\hline Shelfmark & $\begin{array}{l}\text { Catalog pp. } \\
\text { (volume. } \\
\text { pages) }\end{array}$ & $\begin{array}{l}\text { Wright's } \\
\text { Numeration }\end{array}$ & Wright's Dating \\
\hline Add. 14,445 & $1.26-7$ & XLI & 532 \\
\hline Add. 14,446 & 1.98 & CLV & Approx. 6c-7c \\
\hline Add. 14,447 & 1.98 & CLVI & Approx. 10c \\
\hline Add. 14,448 & $1.41-42$ & LXIV & 699-700 \\
\hline Add. 14,449 & $1.46-47$ & LXIX & Approx. 6c-7c \\
\hline Add. 14,450 & 1.55 & LXXIX & Approx. 7c \\
\hline Add. 14,451 & $1.62-63$ & LXXXVIII & Approx. 8c \\
\hline Add. 14,451 & $1.73-75$ & CXIX & Approx. 5c (late) \\
\hline Add. 14,452 & $1.49-50$ & LXXIV & Approx. 6c-7c \\
\hline Add. 14,453 & $1.44-45$ & LXVI & Approx. 5c-6c \\
\hline Add. 14,454 & 1.62 & LXXXVII & Approx. 6c-7c \\
\hline Add. 14,455 & 1.45 & LXVII & Approx. 6c \\
\hline Add. 14,456 & $1.55-57$ & LXXX & Approx. 8c \\
\hline Add. 14,457 & 1.47 & LXX & Approx. 6c-7c \\
\hline Add. 14,458 & $1.47-48$ & LXXI & Approx. 6c-7c \\
\hline $\begin{array}{l}\text { Add. 14,459, } \\
\text { foll. 1-66 }\end{array}$ & 1.64 & XC & Approx. 5c \\
\hline $\begin{array}{l}\text { Add. 14,459, } \\
\text { foll. 67-169 }\end{array}$ & $1.67-68$ & CI & 530-540 \\
\hline Add. 14,460 & $1.52-53$ & LXXVI & 600 \\
\hline $\begin{array}{l}\text { Add. 14,461, } \\
\text { foll. 1-107 }\end{array}$ & $1.65-66$ & XCIV & Approx. 6c \\
\hline $\begin{array}{l}\text { Add. 14,461, } \\
\text { foll. 108-212 }\end{array}$ & 1.68 & CII & Approx. 9c-10c \\
\hline Add. 14,462 & $1.64-65$ & XCII & Approx. 12c \\
\hline Add. 14,463 & $1.54-55$ & LXXVIII \\
\hline Add. 14,464 & $1.70-71$ & CX & Approx. 7c \\
\hline Add. 14,465 & $1.60-61$ & LXXXV & Approx. 6c \\
\hline
\end{tabular}




\begin{tabular}{|c|c|c|c|}
\hline Shelfmark & $\begin{array}{l}\text { Catalog pp. } \\
\text { (volume. } \\
\text { pages) }\end{array}$ & $\begin{array}{l}\text { Wright's } \\
\text { Numeration }\end{array}$ & Wright's Dating \\
\hline $\begin{array}{l}\text { Add. 14,466, } \\
\text { foll. 1-10 }\end{array}$ & 1.69 & CVI & Approx. 12c \\
\hline $\begin{array}{l}\text { Add. 14,466, } \\
\text { foll. 11-17 }\end{array}$ & 1.67 & $\mathrm{C}$ & Approx. 10c-11c \\
\hline $\begin{array}{l}\text { Add. 14,466, } \\
\text { foll. 18-42 }\end{array}$ & 1.73 & CXVIII & Approx. 11c-12c \\
\hline $\begin{array}{l}\text { Add. 14,466, } \\
\text { foll. 43-59 }\end{array}$ & $2.836-837$ & DCCCXX & Approx. 10c-11c \\
\hline Add. 14,467 & $1.66-67$ & XCVII & $\begin{array}{l}\text { Approx. } \\
\text { (early) }\end{array}$ \\
\hline $\begin{array}{l}\text { Add. 14,468, } \\
\text { foll. 1-20 }\end{array}$ & $1.92-93$ & CXLIII & Approx. 7c \\
\hline $\begin{array}{l}\text { Add. 14,468, } \\
\text { foll. } 21-33\end{array}$ & 1.93 & CXLVI & Approx. 10c-11c \\
\hline Add. 14,469 & $1.75-76$ & CXX & 936 \\
\hline Add. 14,470 & $1.40-41$ & LXIII & Approx. 5c-6c, 9c \\
\hline Add. 14,471 & $1.53-54$ & LXXVII & 615 \\
\hline Add. 14,472 & $1.81-82$ & CXXVIII & Approx. 6c-7c \\
\hline $\begin{array}{l}\text { Add. 14,473, } \\
\text { foll. 1-139 }\end{array}$ & $1.79-80$ & CXXV & Approx. 6c \\
\hline $\begin{array}{l}\text { Add. 14,473, } \\
\text { foll. 140-148 }\end{array}$ & $1.83-84$ & CXXXII & Approx. 11c \\
\hline Add. 14,474 & $1.76-77$ & CXXI & Approx. 9c \\
\hline Add. 14,475 & $1.86-87$ & CXXXVI & Approx. 6c \\
\hline Add. 14,476 & 1.84 & CXXXIII & Approx. 5c-6c \\
\hline Add. 14,477 & $1.88-89$ & CXXXVIII & Approx. 6c-7c \\
\hline Add. 14,478 & $1.90-92$ & CXLI & 622 \\
\hline Add. 14,479 & 1.86 & CXXXV & 534 \\
\hline Add. 14,480 & $1.84-85$ & CXXXIV & Approx. 5c-6c \\
\hline Add. 14,481 & 1.90 & CXXXIX & Approx. 6c-7c \\
\hline
\end{tabular}




\begin{tabular}{|l|l|l|l|}
\hline Shelfmark & $\begin{array}{l}\text { Catalog pp. } \\
\text { (volume. } \\
\text { pages) }\end{array}$ & $\begin{array}{l}\text { Wright's } \\
\text { Numeration }\end{array}$ & Wright's Dating \\
\hline Add. 14,482 & $1.112-113$ & CLXV & Approx. 11c-12c \\
\hline Add. 14,483 & 2.591 & DCCVI & Approx. 9c \\
\hline $\begin{array}{l}\text { Add. 14,484, } \\
\text { foll. 1-8 }\end{array}$ & 1.99 & CLVIII & Approx. 5c-6c \\
\hline $\begin{array}{l}\text { Add. 14,484, } \\
\text { foll. 12-47 }\end{array}$ & $1.98-99$ & CLVII & Approx. 6c \\
\hline $\begin{array}{l}\text { Add. 14,484, } \\
\text { foll. 134-152 }\end{array}$ & $3.1152-1153$ & $\begin{array}{l}\text { DCCCCLXXXII } \\
\text { I }\end{array}$ & Approx. 6c \\
\hline $\begin{array}{l}\text { Add. 14,484, } \\
\text { foll. 48-133 }\end{array}$ & 3.1152 & DCCCCLXXXII & Approx. 6c \\
\hline $\begin{array}{l}\text { Add. 14,484, } \\
\text { foll. 9-11 }\end{array}$ & $1.99-100$ & CLIX & Approx. 10c-11c \\
\hline Add. 14,485 & $1.146-149$ & CCXX & 824 \\
\hline Add. 14,486 & $1.149-152$ & CCXXI & 824 \\
\hline Add. 14,487 & $1.152-154$ & CCXXII & 824 \\
\hline Add. 14,488 & $1.194-200$ & CCL & $\begin{array}{l}\text { Approx. 11c - } \\
\text { prob.1023 }\end{array}$ \\
\hline Add. 14,489 & $1.200-202$ & CCLI & 1046 \\
\hline Add. 14,490 & $1.159-161$ & CCXXV & 1089 \\
\hline Add. 14,491 & $1.179-181$ & CCXLIV & Approx. 9c-10c \\
\hline Add. 14,492 & $1.178-179$ & CCXLIII & 862 \\
\hline Add. 14,493 & $1.219-223$ & CCLXXXVI & Approx. 10c \\
\hline Add. 14,494 & $1.217-218$ & CCLXXXIV & Approx. 9c-10c \\
\hline Add. 14,495 & $1.227-228$ & CCXCI & Approx. 10c-11c \\
\hline Add. 14,496 & $1.223-225$ & CCLXXXVII & Approx. 10c \\
\hline Add. 14,497 & $1.231-232$ & CCXCVI & Approx. 11c-12c \\
\hline Add. 14,498 & $1.230-231$ & CCXCV & 1133 \\
\hline Add. 14,499 & $1.229-230$ & CCXCIII & Approx. 10c-11c \\
\hline Add. 14,500 & 1.230 & CCXCIV & Approx. 11c \\
\hline
\end{tabular}




\begin{tabular}{|c|c|c|c|}
\hline Shelfmark & $\begin{array}{l}\text { Catalog pp. } \\
\text { (volume. } \\
\text { pages) }\end{array}$ & $\begin{array}{l}\text { Wright's } \\
\text { Numeration }\end{array}$ & Wright's Dating \\
\hline Add. 14,501 & $1.270-271$ & CCCXXIV & Approx. 11c \\
\hline Add. 14,502 & $1.394-395$ & DXV & Approx. 12c \\
\hline Add. 14,503 & $1.254-258$ & CCCXVIII & Approx. 10c-11c \\
\hline Add. 14,504 & $1.280-282$ & CCCXXXVIII & Approx. 9c \\
\hline Add. 14,505 & $1.282-283$ & CCCXXXIX & Approx. 10c \\
\hline $\begin{array}{l}\text { Add. 14,506, } \\
\text { foll, 1-96 }\end{array}$ & 1.368 & CCCCLXIII & Approx. 11c \\
\hline $\begin{array}{l}\text { Add. 14,506, } \\
\text { foll. 111-118 }\end{array}$ & 1.366 & CCCCLV & Approx. 9c \\
\hline $\begin{array}{l}\text { Add. 14,506, } \\
\text { foll. 119-235 }\end{array}$ & $1.247-249$ & CCCX & Approx. 9c-10c \\
\hline $\begin{array}{l}\text { Add. 14,506, } \\
\text { foll. 97-110 }\end{array}$ & 1.367 & CCCCLX & Approx. 11c \\
\hline Add. 14,507 & $1.283-285$ & CCCXL & Approx. 10c-11c \\
\hline Add. 14,508 & $1.324-325$ & CCCCXIV & Approx. 11c-12c \\
\hline Add. 14,509 & $1.271-272$ & CCCXXV & Approx. 11c \\
\hline Add. 14,510 & $1.378-379$ & CCCCLXXXVI & 1056 \\
\hline Add. 14,511 & $1.249-250$ & CCCXI & Approx. 10c \\
\hline Add. 14,512 & $1.250-251$ & CCCXII & Approx. 10c \\
\hline Add. 14,513 & 1.292 & CCCXLVII & Approx. 9c-10c \\
\hline Add. 14,514 & $1.341-343$ & CCCCXXV & Approx. 9c \\
\hline Add. 14,515 & $1.240-243$ & CCCVI & 893 \\
\hline Add. 14,516 & $1.244-246$ & CCCVIII & Approx. 9c \\
\hline Add. 14,517 & $1.385-388$ & DII & Approx. 10c-11c \\
\hline Add. 14,518 & 1.218 & CCLXXXV & Approx. 9c-10c \\
\hline Add. 14,519 & $1.272-274$ & CCCXXVII & Approx. 11c-12c \\
\hline Add. 14,520 & $1.363-365$ & CCCCLI & Approx. 8c-9c \\
\hline Add. 14,521 & 1.384 & CCCCXCIX & Approx. 10c \\
\hline
\end{tabular}




\begin{tabular}{|l|l|l|l|}
\hline Shelfmark & $\begin{array}{l}\text { Catalog pp. } \\
\text { (volume. } \\
\text { pages) }\end{array}$ & $\begin{array}{l}\text { Wright's } \\
\text { Numeration }\end{array}$ & Wright's Dating \\
\hline $\begin{array}{l}\text { Add. 14,522, } \\
\text { fol. 46 }\end{array}$ & 2.450 & DLXX & Approx. 9c-10c \\
\hline $\begin{array}{l}\text { Add. 14,522, } \\
\text { foll. 1-3 }\end{array}$ & 1.324 & CCCCXIII & Approx. 11c \\
\hline $\begin{array}{l}\text { Add. 14,522, } \\
\text { foll. 27-36 }\end{array}$ & 1.233 & CCXCIX & Approx. 10c-11c \\
\hline $\begin{array}{l}\text { Add. 14,522, } \\
\text { foll. 37-45 }\end{array}$ & 2.831 & DCCCXVI & Approx. 10c \\
\hline $\begin{array}{l}\text { Add. 14,522, } \\
\text { foll. 4-26 }\end{array}$ & $1.246-247$ & CCCIX & Approx. 9c \\
\hline $\begin{array}{l}\text { Add. 14,523, } \\
\text { fol. 38 }\end{array}$ & 1.294 & CCCLI & Approx. 10c \\
\hline $\begin{array}{l}\text { Add. 14,523, } \\
\text { fol. 8 }\end{array}$ & 1.204 & CCLVII & Approx. 8c-9c \\
\hline $\begin{array}{l}\text { Add. 14,523, } \\
\text { fol. 9 }\end{array}$ & 1.204 & CCLVIII & Approx. 10c \\
\hline $\begin{array}{l}\text { Add. 14,523, } \\
\text { foll. 1-7 }\end{array}$ & 1.204 & CCLVI & Approx. 8c-9c \\
\hline $\begin{array}{l}\text { Add. 14,523, } \\
\text { foll. 10-13 }\end{array}$ & $1.383-384$ & CCCCXCV & Approx. 8c \\
\hline $\begin{array}{l}\text { Add. 14,523, } \\
\text { foll. 14-35 }\end{array}$ & 1.293 & CCCXLIX & Approx. 9c-10c \\
\hline $\begin{array}{l}\text { Add. 14,523, } \\
\text { foll. 36, 37 }\end{array}$ & $1.292-293$ & CCCXLVIII & Approx. 9c-10c \\
\hline $\begin{array}{l}\text { Add. 14,523, } \\
\text { foll. 39-46 }\end{array}$ & 1.388 & DIV & Approx. 10c-11c \\
\hline $\begin{array}{l}\text { Add. 14,523, } \\
\text { foll. 47, 48 }\end{array}$ & 1.349 & CCCCXXXIV & Approx. 11c \\
\hline $\begin{array}{l}\text { Add. 14,523, } \\
\text { foll. 49-56 }\end{array}$ & 2.493 & DCXXIV & Approx. 10c \\
\hline $\begin{array}{l}\text { Add. 14,524, } \\
\text { fol. 1 }\end{array}$ & 1.204 & CCLIX & Approx. 10c \\
\hline Add. 14,524, & 1.204 & CCLX & \\
\hline
\end{tabular}




\begin{tabular}{|c|c|c|c|}
\hline Shelfmark & $\begin{array}{l}\text { Catalog pp. } \\
\text { (volume. } \\
\text { pages) }\end{array}$ & $\begin{array}{l}\text { Wright's } \\
\text { Numeration }\end{array}$ & Wright's Dating \\
\hline \multicolumn{4}{|l|}{ fol. 2} \\
\hline $\begin{array}{l}\text { Add. 14,524, } \\
\text { foll. 12-21 }\end{array}$ & 1.349 & CCCCXXXII & Approx. 11c \\
\hline $\begin{array}{l}\text { Add. 14,524, } \\
\text { foll. } 22-29\end{array}$ & 1.349 & CCCCXXXIII & Approx. 11c \\
\hline $\begin{array}{l}\text { Add. 14,524, } \\
\text { foll. 3-6 }\end{array}$ & 1.72 & CXVI & Approx. 11c-12c \\
\hline $\begin{array}{l}\text { Add. 14,524, } \\
\text { foll. } 30-47\end{array}$ & 1.357 & CCCCXLVI & Approx. 13c \\
\hline $\begin{array}{l}\text { Add. 14,524, } \\
\text { foll. 48-67 }\end{array}$ & 1.350 & CCCCXXXV & Approx. 11c-12c \\
\hline $\begin{array}{l}\text { Add. 14,524, } \\
\text { foll. 7-11 }\end{array}$ & 1.143 & CCXI & Approx. 13c \\
\hline $\begin{array}{l}\text { Add. 14,525, } \\
\text { foll. 1-10 }\end{array}$ & $1.393-394$ & DXIV & Approx. 9c-10c \\
\hline $\begin{array}{l}\text { Add. 14,525, } \\
\text { foll. 11-24 }\end{array}$ & $1.302-303$ & CCCLXIX & Approx. 10c-11c \\
\hline $\begin{array}{l}\text { Add. 14,525, } \\
\text { foll. } 25-27\end{array}$ & 1.254 & CCCXV & Approx. 10c-11c \\
\hline $\begin{array}{l}\text { Add. 14,525, } \\
\text { foll. } 28-45\end{array}$ & 1.254 & CCCXVI & Approx. 10c-11c \\
\hline $\begin{array}{l}\text { Add. 14,525, } \\
\text { foll. 46-55 }\end{array}$ & 1.254 & CCCXVII & Approx. 10c-11c \\
\hline $\begin{array}{l}\text { Add. 14,525, } \\
\text { foll. } 56-75\end{array}$ & 1.225 & CCLXXXVIII & Approx. 10c \\
\hline $\begin{array}{l}\text { Add. 14,526, } \\
\text { foll. 1-39 }\end{array}$ & $2.1033-1036$ & DCCCCVII & Approx. 7c \\
\hline $\begin{array}{l}\text { Add. 14,526, } \\
\text { foll. 40-47 }\end{array}$ & 2.1036 & DCCCCVIII & Approx. 8c \\
\hline Add. 14,527 & $2.1036-1037$ & DCCCCIX & Approx. 11c \\
\hline $\begin{array}{l}\text { Add. 14,528, } \\
\text { foll. 1-151 }\end{array}$ & $2.1030-1033$ & DCCCCVI & $\begin{array}{l}\text { Approx. 6c (early) } \\
\text { (501?) }\end{array}$ \\
\hline
\end{tabular}




\begin{tabular}{|c|c|c|c|}
\hline Shelfmark & $\begin{array}{l}\text { Catalog pp. } \\
\text { (volume. } \\
\text { pages) }\end{array}$ & $\begin{array}{l}\text { Wright's } \\
\text { Numeration }\end{array}$ & Wright's Dating \\
\hline $\begin{array}{l}\text { Add. 14,528, } \\
\text { foll. 152-228 }\end{array}$ & $1.176-177$ & CCXXXIX & Approx. 6c \\
\hline Add. 14,529 & $2.917-921$ & DCCCLVI & Approx. 7c-8c \\
\hline Add. 14,530 & $2.1027-1030$ & DCCCCV & 535 \\
\hline Add. 14,531 & $2.738-740$ & DCCLXIX & Approx. 7c-8c \\
\hline Add. 14,532 & $2.955-967$ & DCCCLVIII & Approx. 8c \\
\hline Add. 14,533 & $2.967-976$ & DCCCLIX & Approx. 8c-9c \\
\hline Add. 14,534 & $2.526-527$ & DCLXXV & Approx. 6c \\
\hline Add. 14,535 & $2.796-799$ & DCCXCVIII & Approx. 9c \\
\hline Add. 14,536 & $2.742-744$ & DCCLXXI & Approx. 8c \\
\hline Add. 14,537 & $2.1017-1018$ & DCCCLXVIII & Approx. 7c-8c \\
\hline Add. 14,538 & $2.1003-1008$ & DCCCLXIII & Approx. 10c \\
\hline Add. 14,539 & 2.499 & DCXXVII & Approx. 9c \\
\hline Add. 14,540 & $2.499-500$ & DCXXVIII & Approx. 9c \\
\hline $\begin{array}{l}\text { Add. 14,541, } \\
\text { fol. } 52\end{array}$ & 3.1040 & DCCCCXII & Approx. 9c \\
\hline $\begin{array}{l}\text { Add. 14,541, } \\
\text { foll. 1-38 }\end{array}$ & $2.501-502$ & DCXXX & Approx. 9c \\
\hline $\begin{array}{l}\text { Add. 14,541, } \\
\text { foll. 39-49 }\end{array}$ & $2.585-586$ & DCXCIX & Approx. 8c-9c \\
\hline $\begin{array}{l}\text { Add. } 14,541 \text {, } \\
\text { foll. } 50,51\end{array}$ & 2.450 & DLXIX & Approx. 6c-7c \\
\hline Add. 14,542 & $2.416-418$ & DXLVII & 509 \\
\hline Add. 14,543 & $2.419-420$ & $\mathrm{DL}$ & Approx. 6c \\
\hline Add. 14,544 & $2.421-422$ & DLII & Approx. 5c-6c \\
\hline Add. 14,545 & 2.422 & DLIII & Approx. 6c \\
\hline Add. 14,546 & $2.718-719$ & DCCLVII & Approx. 7c \\
\hline Add. 14,547 & $2.431-434$ & DLVII & Approx. 9c \\
\hline Add. 14,548 & $2.434-436$ & DLVIII & 790 \\
\hline
\end{tabular}




\begin{tabular}{|c|c|c|c|}
\hline Shelfmark & $\begin{array}{l}\text { Catalog pp. } \\
\text { (volume. } \\
\text { pages) }\end{array}$ & $\begin{array}{l}\text { Wright's } \\
\text { Numeration }\end{array}$ & Wright's Dating \\
\hline Add. 14,549 & $2.428-431$ & DLVI & Approx. 8c-9c \\
\hline Add. 14,550 & $2.443-444$ & DLXIV & Approx. 6c \\
\hline Add. 14,551 & 2.485 & DCXI & Approx. 8c \\
\hline Add. 14,552 & $2.485-486$ & DCXII & Approx. 7c-8c \\
\hline Add. 14,553 & $2.489-490$ & DCXVIII & Approx. 6c-7c \\
\hline Add. 14,554 & 2.492 & DCXXI & Approx. 9c \\
\hline Add. 14,555 & $2.483-484$ & DCIX & Approx. 6c-7c \\
\hline Add. 14,556 & $2.487-488$ & DCXIV & Approx. 6c-7c \\
\hline Add. 14,557 & $2.719-721$ & DCCLVIII & Approx. 7c \\
\hline Add. 14,558 & $2.466-467$ & DLXXXIII & 557 \\
\hline Add. 14,559 & $2.468-269$ & DLXXXV & Approx. 6c \\
\hline Add. 14,560 & 2.467 & DLXXXIV & Approx. 6c \\
\hline Add. 14,561 & 2.469 & DLXXXVI & Approx. 6c-7c \\
\hline Add. 14,563 & 2.471 & DLXXXIX & Approx. 7c-8c \\
\hline Add. 14,564 & $2.473-474$ & DXCI & Approx. 6c-7c \\
\hline Add. 14,565 & $2.475-476$ & DXCIII & Approx. 6c-7c \\
\hline $\begin{array}{l}\text { Add. 14,566, } \\
\text { foll. 1-28 }\end{array}$ & 2.476 & DXCIV & Approx. 6c \\
\hline $\begin{array}{l}\text { Add. 14,566, } \\
\text { foll. 29-112 }\end{array}$ & $2.476-477$ & DXCV & Approx. 6c-7c \\
\hline Add. 14,567 & $2.478-479$ & DXCVII & Approx. 6c \\
\hline Add. 14,568 & $2.405-406$ & DXXXI & 599 \\
\hline Add. 14,569 & 2.406 & DXXXII & Approx. 8c \\
\hline Add. 14,570 & $2.406-407$ & DXXXIII & Approx. 5c-6c \\
\hline Add. 14,571 & $2.410-413$ & DXXXIX & 519 \\
\hline Add. 14,572 & 2.410 & DXXXVIII & Approx. 6c \\
\hline Add. 14,573 & $2.413-414$ & DXL & Approx. 6c \\
\hline Add. 14,574, & $2.407-408$ & DXXXV & Approx. 6c \\
\hline
\end{tabular}




\begin{tabular}{|l|l|l|l|}
\hline Shelfmark & $\begin{array}{l}\text { Catalog pp. } \\
\text { (volume. } \\
\text { pages) }\end{array}$ & $\begin{array}{l}\text { Wright's } \\
\text { Numeration }\end{array}$ & Wright's Dating \\
\hline foll. 1-19 & & & \\
\hline $\begin{array}{l}\text { Add. 14,574, } \\
\text { foll. 20-33 }\end{array}$ & $2.408-409$ & DXXXVI & Approx. 5c-6c \\
\hline $\begin{array}{l}\text { Add. 14,574, } \\
\text { foll. 34-40 }\end{array}$ & $2.508-509$ & DCXL & Approx. 6c-7c \\
\hline Add. 14,575 & $2.461-462$ & DLXXVI & Approx. 7c \\
\hline Add. 14,576 & $2.462-464$ & DLXXVII & Approx. 8c \\
\hline Add. 14,577 & $2.784-788$ & DCCXCIII & Approx. 9c \\
\hline Add. 14,578 & $2.445-449$ & DLXVII & Approx. 6c-7c \\
\hline Add. 14,579 & $2.815-818$ & DCCCVIII & 913 \\
\hline Add. 14,580 & $2.767-769$ & DCCLXXXIII & 866 \\
\hline Add. 14,581 & $2.655-657$ & DCCXXXIV & Approx. 6c \\
\hline Add. 14,582 & $2.692-696$ & DCCLII & 816 \\
\hline Add. 14,583 & $3.1076-1078$ & DCCCCXXIX & Approx. 11c \\
\hline Add. 14,584 & $2.506-507$ & DCXXXVIII & Approx. 6c-7c \\
\hline Add. 14,585 & $2.502-503$ & DCXXXI & Approx. 7c \\
\hline Add. 14,586 & 2.503 & DCXXXIII & Approx. 7c-8c \\
\hline Add. 14,587 & $2.517-524$ & DCLXXII & 603 \\
\hline Add. 14,588 & $2.806-807$ & DCCCIII & Approx. 9c-10c \\
\hline Add. 14,589 & $2.858-859$ & DCCCXXX & Approx. 11c-12c \\
\hline Add. 14,590 & $2.752-754$ & DCCLXXVII & Approx. 8c-9c \\
\hline Add. 14,591 & $2.669-673$ & DCCXL & Approx. 6c \\
\hline Add. 14,592 & $2.684-690$ & DCCXLVIII & Approx. 6c-7c \\
\hline Add. 14,593 & $2.590-591$ & DCCIV & 817 \\
\hline Add. 14,594 & $2.618-620$ & DCCXIX & Approx. 9c-10c \\
\hline Add. 14,595 & $2.530-531$ & DCLXXVIII & Approx. 6c-7c \\
\hline Add. 14,596 & $2.531-532$ & DCLXXX & Approx. 6c-7c \\
\hline Add. 14,597 & $2.648-651$ & DCCXXX & 569 \\
\hline
\end{tabular}




\begin{tabular}{|c|c|c|c|}
\hline Shelfmark & $\begin{array}{l}\text { Catalog pp. } \\
\text { (volume. } \\
\text { pages) }\end{array}$ & $\begin{array}{l}\text { Wright's } \\
\text { Numeration }\end{array}$ & Wright's Dating \\
\hline Add. 14,598 & $2.731-732$ & DCCLXIV & Approx. 7c, 9c \\
\hline Add. 14,599 & $2.546-548$ & DCLXXXVI & 569 \\
\hline Add. 14,600 & $2.565-569$ & DCXCIII & Approx. 8c \\
\hline Add. 14,601 & $2.789-793$ & DCCXCV & Approx. 9c \\
\hline Add. 14,602 & $2.701-715$ & DCCLIV & Approx. 6c-7c \\
\hline Add. 14,603 & $2.586-587$ & DCC & Approx. 7c \\
\hline Add. 14,604 & $2.724-726$ & DCCLXI & Approx. 7c \\
\hline Add. 14,605 & $2.715-717$ & DCCLV & 653 \\
\hline Add. 14,606 & $2.744-745$ & DCCLXXII & Approx. 8c \\
\hline Add. 14,607 & $2.683-684$ & DCCXLVII & Approx. 6c-7c \\
\hline $\begin{array}{l}\text { Add. 14,608, } \\
\text { foll. 1-97 }\end{array}$ & $2.733-734$ & DCCLXVI & Approx. 7c-8c \\
\hline $\begin{array}{l}\text { Add. 14,608, } \\
\text { foll. 98-124 }\end{array}$ & $2.723-724$ & DCCLX & Approx. 7c \\
\hline Add. 14,609 & $3.1088-1090$ & DCCCCXLI & Approx. 6c (587) \\
\hline Add. 14,610 & $2.638-639$ & DCCXXVIII & Approx. 6c (551) \\
\hline Add. 14,611 & $2.824-827$ & DCCCXIII & Approx. 10c \\
\hline Add. 14,612 & $2.696-701$ & DCCLIII & Approx. 6c-7c \\
\hline Add. 14,613 & $2.810-815$ & DCCCVI & Approx. 9c-10c \\
\hline $\begin{array}{l}\text { Add. 14,614, } \\
\text { foll. 1-79 }\end{array}$ & $2.832-833$ & DCCCXVII & Approx. 10c \\
\hline $\begin{array}{l}\text { Add. 14,614, } \\
\text { foll. } 80-127\end{array}$ & $2.745-746$ & DCCLXXIII & Approx. 8c \\
\hline Add. 14,615 & $2.840-842$ & DCCCXXIV & Approx. 10c-11c \\
\hline Add. 14,616 & $2.678-680$ & DCCXLIV & Approx. 6c-7c \\
\hline Add. 14,617 & $2.740-742$ & DCCLXX & Approx. 7c-8c \\
\hline Add. 14,618 & $2.736-738$ & DCCLXVIII & Approx. 7c-8c \\
\hline Add. 14,619 & $2.401-403$ & DXXVIII & Approx. 6c \\
\hline Add. 14,620 & $2.800-803$ & DCCC & Approx. 9c \\
\hline
\end{tabular}




\begin{tabular}{|l|l|l|l|}
\hline Shelfmark & $\begin{array}{l}\text { Catalog pp. } \\
\text { (volume. } \\
\text { pages) }\end{array}$ & $\begin{array}{l}\text { Wright's } \\
\text { Numeration }\end{array}$ & Wright's Dating \\
\hline Add. 14,621 & $2.756-759$ & DCCLXXIX & 802 \\
\hline Add. 14,622 & $3.1150-1151$ & $\begin{array}{l}\text { DCCCCLXXVII } \\
\text { I }\end{array}$ & Approx. 9c \\
\hline Add. 14,623 & $2.762-766$ & DCCLXXXI & 823 \\
\hline Add. 14,624 & $2.781-782$ & DCCXCI & Approx. 9c \\
\hline Add. 14,625 & 2.532 & DCLXXXI & Approx. 10c \\
\hline Add. 14,626 & $3.1087-1088$ & DCCCCXL & Approx. 6c \\
\hline Add. 14,627 & 2.415 & DXLII & Approx. 6c-7c \\
\hline $\begin{array}{l}\text { Add. 14,628, } \\
\text { foll. 1-8 }\end{array}$ & $2.1021-1022$ & DCCCLXXVI & Approx. 11c \\
\hline $\begin{array}{l}\text { Add. 14,628, } \\
\text { foll. 9-20 }\end{array}$ & $2.532-533$ & DCLXXXII & Approx. 6c-7c \\
\hline $\begin{array}{l}\text { Add. 14,629, } \\
\text { foll. 1-24 }\end{array}$ & $2.754-756$ & DCCLXXVIII & Approx. 8c-9c \\
\hline $\begin{array}{l}\text { Add. 14,629, } \\
\text { foll. 25-31 }\end{array}$ & 3.1111 & DCCCCLI & Approx. 9c \\
\hline $\begin{array}{l}\text { Add. 14,630, } \\
\text { fol. 28 }\end{array}$ & 2.512 & DCXLVIII & Approx. 7c \\
\hline $\begin{array}{l}\text { Add. 14,630, } \\
\text { foll. 1-27 }\end{array}$ & $2.818-819$ & DCCCX & Approx. 10c \\
\hline $\begin{array}{l}\text { Add. 14,630, } \\
\text { foll. 29-41 }\end{array}$ & 2.481 & DCIII & Approx. 9c \\
\hline $\begin{array}{l}\text { Add. 14,631, } \\
\text { foll. 1-16 }\end{array}$ & 3.1149 & DCCCCLXXII & Approx. 10c \\
\hline $\begin{array}{l}\text { Add. 14,631, } \\
\text { foll. 17-44 }\end{array}$ & 3.1080 & DCCCCXXXIII & Approx. 9c-10c \\
\hline $\begin{array}{l}\text { Add. 14,631, } \\
\text { foll. 45-53 }\end{array}$ & 2.815 & DCCCVII & Approx. 9c-10c \\
\hline Add. 14,632 & $2.576-581$ & DCXCV & Approx. 10c \\
\hline Add. 14,633 & $2.569-576$ & DCXCIV & Approx. 10c \\
\hline Add. 14,634, & 2.515 & DCLVIII & Approx. 9c-10c \\
\hline
\end{tabular}




\begin{tabular}{|c|c|c|c|}
\hline Shelfmark & $\begin{array}{l}\text { Catalog pp. } \\
\text { (volume. } \\
\text { pages) }\end{array}$ & $\begin{array}{l}\text { Wright's } \\
\text { Numeration }\end{array}$ & Wright's Dating \\
\hline \multicolumn{4}{|l|}{ foll. 1,2} \\
\hline $\begin{array}{l}\text { Add. 14,634, } \\
\text { foll. 3-49 }\end{array}$ & $2.514-515$ & DCLVI & Approx. 9c \\
\hline $\begin{array}{l}\text { Add. } 14,634 \text {, } \\
\text { foll. } 50,51\end{array}$ & 2.513 & DCLIII & Approx. 8c-9c \\
\hline $\begin{array}{l}\text { Add. 14,634, } \\
\text { foll. } 52-57\end{array}$ & $2.422-423$ & DLIV & Approx. 9c \\
\hline $\begin{array}{l}\text { Add. 14,635, } \\
\text { foll. 1-4 }\end{array}$ & 2.445 & DLXV & Approx. 6c \\
\hline $\begin{array}{l}\text { Add. 14,635, } \\
\text { foll. 16-18 }\end{array}$ & 2.414 & DXLI & Approx. 6c (555) \\
\hline $\begin{array}{l}\text { Add. 14,635, } \\
\text { foll. } 19,20\end{array}$ & 2.419 & DXLIX & Approx. 8c-9c \\
\hline $\begin{array}{l}\text { Add. 14,635, } \\
\text { foll. 21-24 }\end{array}$ & 1.176 & CCXXXVII & Approx. 11c \\
\hline $\begin{array}{l}\text { Add. 14,635, } \\
\text { foll. 5-15 }\end{array}$ & $2.449-450$ & DLXVIII & Approx. 6c \\
\hline $\begin{array}{l}\text { Add. 14,636, } \\
\text { foll. 1-56 }\end{array}$ & 1.398 & DXXII & Approx. 9c-10c \\
\hline $\begin{array}{l}\text { Add. 14,636, } \\
\text { foll. } 57-76\end{array}$ & 2.1019 & DCCCLXXIII & Approx. 10c \\
\hline $\begin{array}{l}\text { Add. 14,636, } \\
\text { foll. } 77-82\end{array}$ & 2.1020 & DCCCLXXIV & Approx. 10c \\
\hline Add. 14,637 & $2.833-834$ & DCCCXVIII & Approx. 7c-8c \\
\hline $\begin{array}{l}\text { Add. 14,638, } \\
\text { foll. 1-18 }\end{array}$ & $1.398-399$ & DXXIII & Approx. 9c-10c \\
\hline $\begin{array}{l}\text { Add. 14,638, } \\
\text { foll. 19-26 }\end{array}$ & 1.399 & DXXIV & Approx. 10c \\
\hline Add. 14,639 & $3.1039-1040$ & DCCCCXI & Approx. 6c \\
\hline Add. 14,640 & $3.1061-1062$ & DCCCCXX & Approx. 7c \\
\hline Add. 14,641 & $3.1042-1046$ & DCCCCXVIII & Approx. 6c \\
\hline Add. 14,642 & $3.1041-1042$ & DCCCCXVI & Approx. 10c \\
\hline
\end{tabular}




\begin{tabular}{|c|c|c|c|}
\hline Shelfmark & $\begin{array}{l}\text { Catalog pp. } \\
\text { (volume. } \\
\text { pages) }\end{array}$ & \begin{tabular}{|l|} 
Wright's \\
Numeration
\end{tabular} & Wright's Dating \\
\hline Add. 14,643 & $3.1040-1041$ & DCCCCXIII & Approx. 8c (mid) \\
\hline Add. 14,644 & $3.1083-1086$ & DCCCCXXXVI & Approx. 5c-6c \\
\hline Add. 14,645 & $3.1111-1116$ & DCCCCLII & 936 \\
\hline $\begin{array}{l}\text { Add. 14,646, } \\
\text { foll. 1-133 }\end{array}$ & $3.1086-1087$ & DCCCCXXXVII & Approx. 6c \\
\hline $\begin{array}{l}\text { Add. 14,646, } \\
\text { foll. 134-194 }\end{array}$ & 3.1087 & DCCCCXXXIX & Approx. 6c \\
\hline Add. 14,647 & $3.1094-1100$ & DCCCCXLV & 688 \\
\hline Add. 14,648 & $3.1091-1092$ & DCCCCXLIII & Approx. 6c \\
\hline $\begin{array}{l}\text { Add. 14,649, } \\
\text { foll. 1-179 }\end{array}$ & $3.1108-1111$ & DCCCCL & Approx. 9c \\
\hline $\begin{array}{l}\text { Add. 14,649, } \\
\text { foll. 180-205 }\end{array}$ & 2.533 & DCLXXXIII & Approx. 9c \\
\hline Add. 14,650 & $3.1103-1107$ & DCCCCXLIX & Approx. 6c-7c \\
\hline Add. 14,651 & $3.1101-1103$ & DCCCCXLVIII & 850 \\
\hline Add. 14,652 & $2.651-652$ & DCCXXXI & Approx. 6c \\
\hline Add. 14,653 & $2.807-809$ & DCCCIV & Approx. 9c-10c \\
\hline Add. 14,654 & $3.1081-1083$ & DCCCCXXXV & Approx. 5c-6c \\
\hline Add. 14,655 & $3.1119-1120$ & DCCCCLVII & Approx. 11c \\
\hline Add. 14,656 & 2.819 & DCCCXI & Approx. 10c \\
\hline Add. 14,657 & 3.1150 & DCCCCLXXIV & Approx. 9c \\
\hline Add. 14,658 & $3.1154-1160$ & $\begin{array}{l}\text { DCCCCLXXXV } \\
\text { II }\end{array}$ & Approx. 7c \\
\hline Add. 14,660 & $3.1160-1162$ & $\begin{array}{l}\text { DCCCCLXXXV } \\
\text { III }\end{array}$ & Approx. 9c-10c \\
\hline Add. 14,661 & 3.1187 & MIV & Approx. 6c-7c \\
\hline Add. 14,662 & 3.1189 & MVI & Approx. 9c \\
\hline Add. 14,663 & $2.691-692$ & DCCLI & Approx. 6c-7c \\
\hline $\begin{array}{l}\text { Add. 14,664, } \\
\text { fol. } 34\end{array}$ & 1.378 & CCCCLXXXV & Approx. 12c-13c \\
\hline
\end{tabular}




\begin{tabular}{|c|c|c|c|}
\hline Shelfmark & $\begin{array}{l}\text { Catalog pp. } \\
\text { (volume. } \\
\text { pages) }\end{array}$ & $\begin{array}{l}\text { Wright's } \\
\text { Numeration }\end{array}$ & Wright's Dating \\
\hline $\begin{array}{l}\text { Add. 14,664, } \\
\text { foll, 1-17, 20, } \\
21\end{array}$ & 1.203 & CCLIV & Approx. 10c-11c \\
\hline $\begin{array}{l}\text { Add. 14,664, } \\
\text { foll. 18, 19, } \\
30-33\end{array}$ & 1.389 & DVI & Approx. 12c-13c \\
\hline $\begin{array}{l}\text { Add. 14,664, } \\
\text { foll. } 22-29\end{array}$ & 1.39 & LXII & Approx. 10c-11c \\
\hline $\begin{array}{l}\text { Add. 14,665, } \\
\text { fol. } 25\end{array}$ & 1.389 & DVIII & Approx. 10c \\
\hline $\begin{array}{l}\text { Add. 14,665, } \\
\text { fol. } 28\end{array}$ & $3.1172-1173$ & DCCCCXCVII & Approx. (9c-10c) \\
\hline $\begin{array}{l}\text { Add. 14,665, } \\
\text { foll. 1-7 }\end{array}$ & $3.1118-1119$ & DCCCCLV & Approx. 10c-11c \\
\hline $\begin{array}{l}\text { Add. 14,665, } \\
\text { foll. 10-20 }\end{array}$ & 2.837 & DCCCXXI & Approx. 6c-7c \\
\hline $\begin{array}{l}\text { Add. 14,665, } \\
\text { foll. 21-24 }\end{array}$ & 1.389 & DVII & Approx. 12c-13c \\
\hline $\begin{array}{l}\text { Add. 14,665, } \\
\text { foll. } 26,27\end{array}$ & 2.1022 & DCCCLXXVII & $\begin{array}{l}\text { Approx. 8c, 12c- } \\
13 \mathrm{c}\end{array}$ \\
\hline $\begin{array}{l}\text { Add. 14,665, } \\
\text { foll. } 8,9\end{array}$ & 3.1151 & DCCCCLXXXI & Approx. 8c \\
\hline $\begin{array}{l}\text { Add. 14,666, } \\
\text { fol. } 1\end{array}$ & 1.17 & XXVIII & Approx. 10c-11c \\
\hline $\begin{array}{l}\text { Add. 14,666, } \\
\text { fol. } 2\end{array}$ & 1.17 & XXVII & Approx. 8c \\
\hline $\begin{array}{l}\text { Add. 14,666, } \\
\text { fol. } 3\end{array}$ & 1.13 & XX & Approx. 6c-7c \\
\hline $\begin{array}{l}\text { Add. 14,666, } \\
\text { fol. } 47\end{array}$ & 1.69 & CVII & Approx. 12c \\
\hline $\begin{array}{l}\text { Add. 14,666, } \\
\text { fol. } 48\end{array}$ & 1.69 & $\mathrm{CV}$ & Approx. 10c \\
\hline Add. 14,666, & 1.92 & CXLII & 682 \\
\hline
\end{tabular}




\begin{tabular}{|l|l|l|l|}
\hline Shelfmark & $\begin{array}{l}\text { Catalog pp. } \\
\text { (volume. } \\
\text { pages) }\end{array}$ & $\begin{array}{l}\text { Wright's } \\
\text { Numeration }\end{array}$ & Wright's Dating \\
\hline fol. 56 & & & \\
\hline $\begin{array}{l}\text { Add. 14,666, } \\
\text { foll. 10-12 }\end{array}$ & 1.123 & CLXXIV & Approx. 9c-10c \\
\hline $\begin{array}{l}\text { Add. 14,666, } \\
\text { foll. 13-32 }\end{array}$ & 1.141 & CCIV & Approx. 12c \\
\hline $\begin{array}{l}\text { Add. 14,666, } \\
\text { foll. 33-36 }\end{array}$ & 1.141 & CCV & Approx. 12c-13c \\
\hline $\begin{array}{l}\text { Add. 14,666, } \\
\text { foll. 37 }\end{array}$ & 1.27 & XLV & Approx. 6c-7c \\
\hline $\begin{array}{l}\text { Add. 14,666, } \\
\text { foll. 38-46 }\end{array}$ & 1.27 & XLIII & Approx. 6c-7c \\
\hline $\begin{array}{l}\text { Add. 14,666, } \\
\text { foll. 4, 5 }\end{array}$ & 1.123 & CLXXII & Approx. 9c \\
\hline $\begin{array}{l}\text { Add. 14,666, } \\
\text { foll. 49, 50 }\end{array}$ & 1.72 & CXV & Approx. 10c-11c \\
\hline $\begin{array}{l}\text { Add. 14,666, } \\
\text { foll. 51-55 }\end{array}$ & 1.93 & CXLIV & Approx. 7c-8c \\
\hline $\begin{array}{l}\text { Add. 14,666, } \\
\text { foll. 57-64 }\end{array}$ & $2.732-733$ & DCCLXV & Approx. 7c-8c \\
\hline $\begin{array}{l}\text { Add. 14,666, } \\
\text { foll. 6-9 }\end{array}$ & 1.123 & CLXXIII & Approx. 9c \\
\hline $\begin{array}{l}\text { Add. 14,667, } \\
\text { fol. 43 }\end{array}$ & 1.312 & CCCXCI & Approx. 12c \\
\hline $\begin{array}{l}\text { Add. 14,667, } \\
\text { fol. 54 }\end{array}$ & 1.366 & CCCCLIV & Approx. 9c \\
\hline $\begin{array}{l}\text { Add. 14,667, } \\
\text { fol. 64 }\end{array}$ & 3.1197 & MXIX & Approx. 10c \\
\hline $\begin{array}{l}\text { Add. 14,667, } \\
\text { foll, 13-16 } \\
\text { foll. 1-12 }\end{array}$ & 1.233 & CCXCVIII & Approx. 9c-10c \\
\hline $\begin{array}{l}\text { Add. 14,667, } \\
\text { foll, 17-28 }\end{array}$ & 1.225 & CCLXXXIX & Approx. 10c-11c \\
\hline $\begin{array}{l}\text { Add. 14,667, } \\
\text { (1.111 }\end{array}$ & CLXII & \\
\hline
\end{tabular}




\begin{tabular}{|c|c|c|c|}
\hline Shelfmark & $\begin{array}{l}\text { Catalog pp. } \\
\text { (volume. } \\
\text { pages) }\end{array}$ & $\begin{array}{l}\text { Wright's } \\
\text { Numeration }\end{array}$ & Wright's Dating \\
\hline $\begin{array}{l}\text { Add. 14,667, } \\
\text { foll. 29-38 }\end{array}$ & $1.228-229$ & CCXCII & Approx. 10c-11c \\
\hline $\begin{array}{l}\text { Add. 14,667, } \\
\text { foll. 39, } 40\end{array}$ & $1.388-389$ & DV & Approx. 10c-11c \\
\hline $\begin{array}{l}\text { Add. 14,667, } \\
\text { foll. 41, } 42\end{array}$ & 1.367 & CCCCLXII & Approx. 11c \\
\hline $\begin{array}{l}\text { Add. 14,667, } \\
\text { foll. } 44,45\end{array}$ & 1.384 & CCCCXCVI & Approx. 8c \\
\hline $\begin{array}{l}\text { Add. 14,667, } \\
\text { foll. 46-49 }\end{array}$ & $1.384-385$ & $\mathrm{DI}$ & Approx. 10c \\
\hline $\begin{array}{l}\text { Add. 14,667, } \\
\text { foll. } 50,51\end{array}$ & $3.1197-1198$ & MXXIV & 1057 \\
\hline $\begin{array}{l}\text { Add. 14,667, } \\
\text { foll. } 52,53\end{array}$ & 1.384 & $\mathrm{D}$ & Approx. 10c \\
\hline $\begin{array}{l}\text { Add. 14,667, } \\
\text { foll. } 55-59\end{array}$ & 1.316 & CCCCIII & Approx. 11c \\
\hline $\begin{array}{l}\text { Add. 14,667, } \\
\text { foll. } 60-63\end{array}$ & 1.295 & CCCLII & Approx. 10c \\
\hline $\begin{array}{l}\text { Add. 14,667, } \\
\text { foll. } 65-69\end{array}$ & 1.295 & CCCLIII & Approx. 10c \\
\hline $\begin{array}{l}\text { Add. } 14,667 \text {, } \\
\text { foll. } 70,71\end{array}$ & 1.254 & CCCXIV & Approx. 10c \\
\hline $\begin{array}{l}\text { Add. 14,667, } \\
\text { foll. } 72,73\end{array}$ & $1.202-203$ & CCLIII & Approx. 11c \\
\hline $\begin{array}{l}\text { Add. 14,667, } \\
\text { foll. } 74,75\end{array}$ & 1.312 & CCCXC & Approx. 11c \\
\hline $\begin{array}{l}\text { Add. 14,667, } \\
\text { foll. } 76-78\end{array}$ & 1.316 & CCCCI & Approx. 10c-11c \\
\hline $\begin{array}{l}\text { Add. 14,668, } \\
\text { fol. } 44\end{array}$ & 2.788 & DCCXCIV & Approx. 9c \\
\hline $\begin{array}{l}\text { Add. 14,668, } \\
\text { fol. } 45\end{array}$ & $2.480-481$ & $\mathrm{DC}$ & Approx. 8c-9c \\
\hline Add. 14,668, & 3.1196 & MXVI & 888 \\
\hline
\end{tabular}




\begin{tabular}{|c|c|c|c|}
\hline Shelfmark & $\begin{array}{l}\text { Catalog pp. } \\
\text { (volume. } \\
\text { pages) }\end{array}$ & $\begin{array}{l}\text { Wright's } \\
\text { Numeration }\end{array}$ & Wright's Dating \\
\hline \multicolumn{4}{|l|}{ fol. 46} \\
\hline $\begin{array}{l}\text { Add. 14,668, } \\
\text { foll. 1-3 }\end{array}$ & 1.10 & XIV & Approx. 9c \\
\hline $\begin{array}{l}\text { Add. 14,668, } \\
\text { foll. 12-19 }\end{array}$ & 1.27 & XLIV & Approx. 7c \\
\hline $\begin{array}{l}\text { Add. 14,668, } \\
\text { foll. } 20-25\end{array}$ & $1.20-21$ & XXXIII & Approx. 8c \\
\hline $\begin{array}{l}\text { Add. 14,668, } \\
\text { foll. 26-29 }\end{array}$ & 1.37 & LVIII & Approx. 8c \\
\hline $\begin{array}{l}\text { Add. 14,668, } \\
\text { foll. } 30,31\end{array}$ & 1.72 & CXIV & Approx. 9c \\
\hline $\begin{array}{l}\text { Add. 14,668, } \\
\text { foll. 32-36 }\end{array}$ & 2.482 & DCVII & Approx. 6c \\
\hline $\begin{array}{l}\text { Add. 14,668, } \\
\text { foll. 37-39 }\end{array}$ & $2.605-606$ & DCCIX & Approx. 9c-10c \\
\hline $\begin{array}{l}\text { Add. 14,668, } \\
\text { foll. 4-11 }\end{array}$ & 1.37 & LIX & Approx. 8c \\
\hline $\begin{array}{l}\text { Add. 14,668, } \\
\text { foll. 40-43 }\end{array}$ & 2.769 & DCCLXXXIV & 866 \\
\hline $\begin{array}{l}\text { Add. 14,669, } \\
\text { fol. } 19\end{array}$ & 2.481 & DCI & Approx. 10c \\
\hline $\begin{array}{l}\text { Add. 14,669, } \\
\text { fol. } 25\end{array}$ & 1.22 & XXXV & Approx. 7c \\
\hline $\begin{array}{l}\text { Add. 14,669, } \\
\text { fol. } 26\end{array}$ & 1.69 & CVIII & Approx. 12c \\
\hline $\begin{array}{l}\text { Add. 14,669, } \\
\text { fol. } 37\end{array}$ & 1.72 & CXIII & Approx. 6c \\
\hline $\begin{array}{l}\text { Add. 14,669, } \\
\text { foll. 1-18 }\end{array}$ & 2.483 & DCVIII & Approx. 5c-6c \\
\hline $\begin{array}{l}\text { Add. 14,669, } \\
\text { foll. 20, } 21\end{array}$ & 1.204 & CCLV & Approx. 6c \\
\hline $\begin{array}{l}\text { Add. 14,669, } \\
\text { foll. } 22\end{array}$ & 1.10 & $\mathrm{XV}$ & Approx. 10c \\
\hline
\end{tabular}




\begin{tabular}{|c|c|c|c|}
\hline Shelfmark & $\begin{array}{l}\text { Catalog pp. } \\
\text { (volume. } \\
\text { pages) }\end{array}$ & $\begin{array}{l}\text { Wright's } \\
\text { Numeration }\end{array}$ & Wright's Dating \\
\hline $\begin{array}{l}\text { Add. 14,669, } \\
\text { foll. } 23,24\end{array}$ & 1.10 & XIII & Approx. 6c \\
\hline $\begin{array}{l}\text { Add. 14,669, } \\
\text { foll. 29-33 }\end{array}$ & $1.68-69$ & CIII & Approx. 6c-7c \\
\hline $\begin{array}{l}\text { Add. 14,669, } \\
\text { foll. 34-36 }\end{array}$ & 1.67 & XCIX & Approx. 6c \\
\hline $\begin{array}{l}\text { Add. 14,669, } \\
\text { foll. 38-56 }\end{array}$ & 1.58 & LXXXII & Approx. 9c \\
\hline $\begin{array}{l}\text { Add. 14,669, } \\
\text { foll. 57-59 }\end{array}$ & 1.90 & CXL & Approx. 6c-7c \\
\hline $\begin{array}{l}\text { Add. 14,670, } \\
\text { fol. } 1\end{array}$ & 2.480 & DXCIX & Approx. 6c-7c \\
\hline $\begin{array}{l}\text { Add. 14,670, } \\
\text { fol. } 14\end{array}$ & 2.464 & DLXXVIII & Approx. 6c \\
\hline $\begin{array}{l}\text { Add. 14,670, } \\
\text { fol. } 38\end{array}$ & 2.512 & DCXLIX & Approx. 7c \\
\hline $\begin{array}{l}\text { Add. 14,670, } \\
\text { fol. } 39\end{array}$ & 2.512 & DCXLVII & Approx. 7c \\
\hline $\begin{array}{l}\text { Add. 14,670, } \\
\text { foll. 15-18 }\end{array}$ & 3.1164 & DCCCCXCII & Approx. 7c \\
\hline $\begin{array}{l}\text { Add. 14,670, } \\
\text { foll. 19-22 }\end{array}$ & $2.690-691$ & DCCL & Approx. 6c-7c \\
\hline $\begin{array}{l}\text { Add. 14,670, } \\
\text { foll. 2-7 }\end{array}$ & $2.588-589$ & DCCII & Approx. 9c \\
\hline $\begin{array}{l}\text { Add. 14,670, } \\
\text { foll. } 23-25\end{array}$ & 3.1153 & $\begin{array}{l}\text { DCCCCLXXXI } \\
\mathrm{V}\end{array}$ & Approx. 6c-7c \\
\hline $\begin{array}{l}\text { Add. 14,670, } \\
\text { foll. 26-29 }\end{array}$ & 2.510 & DCXLIII & Approx. 6c-7c \\
\hline $\begin{array}{l}\text { Add. 14,670, } \\
\text { foll. } 30-35\end{array}$ & 2.510 & DCXLII & Approx. 6c-7c \\
\hline $\begin{array}{l}\text { Add. 14,670, } \\
\text { foll. } 36,37\end{array}$ & 2.511 & DCXLIV & Approx. 6c-7c \\
\hline Add. 14,670, & 2.464 & DLXXIX & Approx. 9c \\
\hline
\end{tabular}




\begin{tabular}{|l|l|l|l|}
\hline Shelfmark & $\begin{array}{l}\text { Catalog pp. } \\
\text { (volume. } \\
\text { pages) }\end{array}$ & $\begin{array}{l}\text { Wright's } \\
\text { Numeration }\end{array}$ & Wright's Dating \\
\hline foll. 8-13 & & & \\
\hline Add. 14,671 & 1.3 & II & Approx. 13c \\
\hline Add. 14,672 & 1.136 & CXCIII & Approx. 13c-14c \\
\hline Add. 14,673 & 1.132 & CLXXXVIII & Approx. 13c \\
\hline $\begin{array}{l}\text { Add. 14,674, } \\
\text { foll. 1-78 }\end{array}$ & $1.127-128$ & CLXXX & Approx. 12c \\
\hline $\begin{array}{l}\text { Add. 14,674, } \\
\text { foll. 79-126 }\end{array}$ & 1.128 & CLXXXI & Approx. 12c \\
\hline Add. 14,675 & $1.129-131$ & CLXXXVI & Approx. 13c \\
\hline $\begin{array}{l}\text { Add. 14,676, } \\
\text { foll. 1-42 }\end{array}$ & 1.128 & CLXXXII & Approx. 12c \\
\hline $\begin{array}{l}\text { Add. 14,676, } \\
\text { foll. 43-86 }\end{array}$ & 3.1074 & DCCCCXXVI & Approx. 13c \\
\hline Add. 14,677 & $1.131-132$ & CLXXXVII & Approx. 13c \\
\hline Add. 14,678 & $1.132-133$ & CLXXXIX & 1237 \\
\hline Add. 14,679 & 2.606 & DCCX & 1102 \\
\hline Add. 14,680 & $1.77-78$ & CXXII & Approx. 12c-13c \\
\hline Add. 14,681 & $1.78-79$ & CXXIII & Approx. 12c-13c \\
\hline Add. 14,682 & $2.608-610$ & DCCXIII & Approx. 10c \\
\hline Add. 14,683 & $2.610-612$ & DCCXIV & Approx. 10c \\
\hline $\begin{array}{l}\text { Add. 14,684, } \\
\text { foll. 1-36 }\end{array}$ & 1.113 & CLXVI & Approx. 12c \\
\hline $\begin{array}{l}\text { Add. 14,684, } \\
\text { foll. 37-117 }\end{array}$ & $1.113-115$ & CLXVII & Approx. 12c-13c \\
\hline Add. 14,685 & $3.1062-1064$ & DCCCCXXI & Approx. 10c-11c \\
\hline Add. 14,686 & $1.169-172$ & CCXXVIII & 1255 \\
\hline Add. 14,687 & $1.172-173$ & CCXXIX & 1256 \\
\hline Add. 14,688 & $1.188-190$ & CCXLVII & Approx. 12c-13c \\
\hline Add. 14,689 & $1.167-169$ & CCXXVII & 1221 \\
\hline
\end{tabular}




\begin{tabular}{|c|c|c|c|}
\hline Shelfmark & $\begin{array}{l}\text { Catalog pp. } \\
\text { (volume. } \\
\text { pages) }\end{array}$ & $\begin{array}{l}\text { Wright's } \\
\text { Numeration }\end{array}$ & Wright's Dating \\
\hline Add. 14,690 & $1.205-207$ & CCLXI & 1182 \\
\hline $\begin{array}{l}\text { Add. 14,691, } \\
\text { foll. 1-109 }\end{array}$ & $1.208-209$ & CCLXIV & 1230 \\
\hline $\begin{array}{l}\text { Add. 14,691, } \\
\text { foll. 110-114 }\end{array}$ & 1.95 & CL & Approx. 13c \\
\hline $\begin{array}{l}\text { Add. 14,692, } \\
\text { foll. 1-24 }\end{array}$ & $1.390-391$ & DXI & Approx. 15c \\
\hline $\begin{array}{l}\text { Add. 14,692, } \\
\text { foll. 25-99 }\end{array}$ & $1.211-212$ & CCLXXIII & 1347 \\
\hline $\begin{array}{l}\text { Add. 14,693, } \\
\text { foll. 1-141 }\end{array}$ & $1.210-211$ & CCLXXII & Approx. 13c-14c \\
\hline $\begin{array}{l}\text { Add. 14,693, } \\
\text { foll. 142-184 }\end{array}$ & 1.212 & CCLXXIV & Approx. 14c \\
\hline $\begin{array}{l}\text { Add. 14,694, } \\
\text { foll. 1-43 }\end{array}$ & 1.209 & CCLXVI & Approx. 13c \\
\hline $\begin{array}{l}\text { Add. 14,694, } \\
\text { foll. 44-106 }\end{array}$ & $1.209-210$ & CCLXVII & Approx. 13c \\
\hline Add. 14,695 & $1.285-286$ & CCCXLI & Approx. 11c-12c \\
\hline Add. 14,696 & $1.289-290$ & CCCXLIV & Approx. 13c \\
\hline Add. 14,697 & $1.286-288$ & CCCXLII & Approx. 12c (late) \\
\hline Add. 14,698 & $1.288-289$ & CCCXLIII & Approx. 12c-13c \\
\hline Add. 14,699 & $1.304-306$ & CCCLXXIV & 1292 \\
\hline Add. 14,700 & 1.306 & CCCLXXV & 1292 \\
\hline Add. 14,701 & $1.278-279$ & CCCXXXV & 1263 \\
\hline Add. 14,702 & $1.309-310$ & CCCLXXXV & Approx. 15c/1489 \\
\hline Add. 14,703 & $1.370-371$ & CCCCLXVII & Approx. 12c-13c \\
\hline Add. 14,704 & $1.312-313$ & CCCXCIII & Approx. 13c \\
\hline Add. 14,705 & $1.181-182$ & CCXLV & Approx. 11c \\
\hline Add. 14,706 & $1.396-397$ & DXX & Approx. 13c \\
\hline Add. 14,707 & $1.277-278$ & CCCXXXIV & Approx. \\
\hline
\end{tabular}




\begin{tabular}{|l|l|l|l|}
\hline Shelfmark & $\begin{array}{l}\text { Catalog pp. } \\
\text { (volume. } \\
\text { pages) }\end{array}$ & $\begin{array}{l}\text { Wright's } \\
\text { Numeration }\end{array}$ & Wright's Dating \\
\hline & & & (early) \\
\hline $\begin{array}{l}\text { Add. 14,708, } \\
\text { foll. 1-51 }\end{array}$ & $1.310-311$ & CCCLXXXVI & Approx. 15c (late) \\
\hline $\begin{array}{l}\text { Add. 14,708, } \\
\text { foll. 122-161 }\end{array}$ & 1.309 & CCCLXXXIV & Approx. 14c \\
\hline $\begin{array}{l}\text { Add. 14,708, } \\
\text { foll. 52-121 }\end{array}$ & $1.308-309$ & CCCLXXXIII & Approx. 14c \\
\hline $\begin{array}{l}\text { Add. 14,709, } \\
\text { foll. 1-71 }\end{array}$ & 1.303 & CCCLXXI & 1336 \\
\hline $\begin{array}{l}\text { Add. 14,709, } \\
\text { foll. 72-74 }\end{array}$ & 1.306 & CCCLXXVII & 1336 \\
\hline $\begin{array}{l}\text { Add. 14,709, } \\
\text { foll. 75-94 }\end{array}$ & $1.175-176$ & CCXXXVI & 1484 \\
\hline Add. 14,710 & 1.325 & CCCCXV & 1259 \\
\hline Add. 14,711 & $1.320-321$ & CCCCIX & 1222 \\
\hline Add. 14,712 & $1.291-292$ & CCCXLVI & Approx. 13c \\
\hline Add. 14,713 & $1.351-352$ & CCCCXXXVII & Approx. 12c-13c \\
\hline Add. 14,714 & $1.346-347$ & CCCCXXX & 1075 \\
\hline $\begin{array}{l}\text { Add. 14,715, } \\
\text { foll. 1-152 }\end{array}$ & $1.379-380$ & CCCCLXXXVII & 1257 \\
\hline $\begin{array}{l}\text { Add. 14,715, } \\
\text { foll. 153-216 }\end{array}$ & $1.233-234$ & CCC & Approx. 13c \\
\hline $\begin{array}{l}\text { Add. 14,715, } \\
\text { foll. 217-241 }\end{array}$ & $1.311-312$ & CCCLXXXIX & Approx. 13c \\
\hline $\begin{array}{l}\text { Add. 14,716, } \\
\text { foll. 1-78 }\end{array}$ & $1.328-329$ & CCCCXX & Approx. 13c \\
\hline $\begin{array}{l}\text { Add. 14,716, } \\
\text { foll. 79-166 }\end{array}$ & $1.374-375$ & CCCCLXX & Approx. 13c \\
\hline $\begin{array}{l}\text { Add. 14,717, } \\
\text { foll. 1-58 }\end{array}$ & $1.395-396$ & DXVIII & Approx. 13c \\
\hline Add. 14,717, & 1.307 & CCCLXXIX & Approx. 13c-14c \\
\hline
\end{tabular}




\begin{tabular}{|c|c|c|c|}
\hline Shelfmark & $\begin{array}{l}\text { Catalog pp. } \\
\text { (volume. } \\
\text { pages) }\end{array}$ & $\begin{array}{l}\text { Wright's } \\
\text { Numeration }\end{array}$ & Wright's Dating \\
\hline \multicolumn{4}{|l|}{ foll. 103-130 } \\
\hline $\begin{array}{l}\text { Add. 14,717, } \\
\text { foll. 59-102 }\end{array}$ & 1.311 & CCCLXXXVII & Approx. 13c \\
\hline Add. 14,718 & $1.298-299$ & CCCLXIII & Approx. 13c \\
\hline Add. 14,719 & $1.275-276$ & CCCXXX & 1184 \\
\hline $\begin{array}{l}\text { Add. 14,720, } \\
\text { foll. 1-111 }\end{array}$ & 1.313 & CCCXCIV & Approx. 13c \\
\hline $\begin{array}{l}\text { Add. 14,720, } \\
\text { foll. 112-135 }\end{array}$ & $1.381-382$ & CCCCXCII & Approx. 14c \\
\hline Add. 14,721 & $1.376-377$ & CCCCLXXII & Approx. 13c \\
\hline $\begin{array}{l}\text { Add. 14,722, } \\
\text { foll. 1-61 }\end{array}$ & $2.1023-1024$ & DCCCLXXX & Approx. 13c \\
\hline $\begin{array}{l}\text { Add. 14,722, } \\
\text { foll. } 62-77\end{array}$ & 1.377 & CCCCLXXIX & Approx. 14c \\
\hline $\begin{array}{l}\text { Add. 14,723, } \\
\text { foll. } 1,2\end{array}$ & 1.377 & CCCCLXXIII & Approx. 13c \\
\hline $\begin{array}{l}\text { Add. 14,723, } \\
\text { foll. 3-65 }\end{array}$ & 1.358 & CCCCXLVIII & Approx. 13c \\
\hline $\begin{array}{l}\text { Add. 14,723, } \\
\text { foll. 66-113 }\end{array}$ & $1.144-145$ & CCXVII & Approx. 13c \\
\hline $\begin{array}{l}\text { Add. 14,724, } \\
\text { foll. 1-113 }\end{array}$ & $1.375-376$ & CCCCLXXI & Approx. 13c \\
\hline $\begin{array}{l}\text { Add. 14,724, } \\
\text { foll. 114-144 }\end{array}$ & $1.380-381$ & CCCCLXXXVIII & Approx. 13c \\
\hline $\begin{array}{l}\text { Add. 14,725, } \\
\text { fol. } 99\end{array}$ & 1.399 & DXXVI & Approx. 10c-11c \\
\hline $\begin{array}{l}\text { Add. 14,725, } \\
\text { foll. 1-95 }\end{array}$ & $2.827-828$ & DCCCXIV & Approx. 10c \\
\hline $\begin{array}{l}\text { Add. } 14,725, \\
\text { foll. } 100-215\end{array}$ & $2.441-443$ & DLXIII & Approx. 10c-11c \\
\hline $\begin{array}{l}\text { Add. 14,725, } \\
\text { foll. 96-98 }\end{array}$ & 1.399 & DXXV & Approx. 10c-11c \\
\hline
\end{tabular}




\begin{tabular}{|l|l|l|l|}
\hline Shelfmark & $\begin{array}{l}\text { Catalog pp. } \\
\text { (volume. } \\
\text { pages) }\end{array}$ & $\begin{array}{l}\text { Wright's } \\
\text { Numeration }\end{array}$ & Wright's Dating \\
\hline $\begin{array}{l}\text { Add. 14,726, } \\
\text { foll. 1-86 }\end{array}$ & $2.828-831$ & DCCCXV & Approx. 10c \\
\hline $\begin{array}{l}\text { Add. 14,726, } \\
\text { foll. 87-128 }\end{array}$ & $2.617-618$ & DCCXVIII & Approx. 10c \\
\hline Add. 14,727 & $2.886-890$ & DCCCXLVIII & Approx. 13c \\
\hline $\begin{array}{l}\text { Add. 14,728, } \\
\text { foll. 1-75 }\end{array}$ & $2.882-883$ & DCCCXLII & Approx. 13c \\
\hline $\begin{array}{l}\text { Add. 14,728, } \\
\text { foll. 138-207 }\end{array}$ & $2.883-884$ & DCCCXLIII & Approx. 13c \\
\hline $\begin{array}{l}\text { Add. 14,728, } \\
\text { foll. 208-238 }\end{array}$ & 2.581 & DCXCVI & Approx. 12c-13c \\
\hline $\begin{array}{l}\text { Add. 14,728, } \\
\text { foll. 239-268 }\end{array}$ & $2.581-584$ & DCXCVII & Approx. 12c-13c \\
\hline $\begin{array}{l}\text { Add. 14,728, } \\
\text { foll. 76-137 }\end{array}$ & 2.876 & DCCCXL & Approx. 12c-13c \\
\hline $\begin{array}{l}\text { Add. 14,729, } \\
\text { foll. 1-115 }\end{array}$ & $2.860-865$ & DCCCXXXII & 1172-3 \\
\hline $\begin{array}{l}\text { Add. 14,729, } \\
\text { foll. 116-123 }\end{array}$ & $2.873-874$ & DCCCXXXVIII & Approx. 12c-13c \\
\hline $\begin{array}{l}\text { Add. 14,729, } \\
\text { foll. 124-192 }\end{array}$ & $2.874-876$ & DCCCXXXIX & Approx. 12c-13c \\
\hline $\begin{array}{l}\text { Add. 14,729, } \\
\text { foll. 193-198 }\end{array}$ & $1.389-390$ & DIX & Approx. 13c \\
\hline $\begin{array}{l}\text { Add. 14,729, } \\
\text { foll. 199-234 }\end{array}$ & $2.584-585$ & DCXCVIII & Approx. 12c-13c \\
\hline $\begin{array}{l}\text { Add. 14,730, } \\
\text { foll. 1-111 }\end{array}$ & $2.865-866$ & DCCCXXXIV & Approx. 12c \\
\hline $\begin{array}{l}\text { Add. 14,730, } \\
\text { foll. 112-164 }\end{array}$ & $3.1122-1123$ & DCCCCLIX & Approx. 12c \\
\hline Add. 14,731 & $2.853-855$ & DCCCXXVII & Approx. 11c \\
\hline fdd. 14,732, 228 & 2.517 & DCLXVIII & Approx. 12c-13c \\
\hline
\end{tabular}




\begin{tabular}{|c|c|c|c|}
\hline Shelfmark & $\begin{array}{l}\text { Catalog pp. } \\
\text { (volume. } \\
\text { pages) }\end{array}$ & $\begin{array}{l}\text { Wright's } \\
\text { Numeration }\end{array}$ & Wright's Dating \\
\hline $\begin{array}{l}\text { Add. 14,732, } \\
\text { foll. 1-227 }\end{array}$ & $3.1141-1146$ & DCCCCLXIII & Approx. 13c \\
\hline $\begin{array}{l}\text { Add. 14,732, } \\
\text { foll. 229-237 }\end{array}$ & 2.867 & DCCCXXXVI & Approx. 12c \\
\hline Add. 14,733 & $3.1139-1140$ & DCCCCLXI & 1199 \\
\hline $\begin{array}{l}\text { Add. 14,734, } \\
\text { foll. 1-176 }\end{array}$ & $3.1147-1148$ & DCCCCLXVII & 1085 \\
\hline $\begin{array}{l}\text { Add. 14,734, } \\
\text { foll. 177-223 }\end{array}$ & 3.1119 & DCCCCLVI & Approx. 11c \\
\hline $\begin{array}{l}\text { Add. 14,735, } \\
\text { foll. } 1-23\end{array}$ & 3.1151 & DCCCCLXXIX & Approx. 13c \\
\hline $\begin{array}{l}\text { Add. } 14,735 \text {, } \\
\text { foll. } 24-50\end{array}$ & 3.1149 & DCCCCLXXI & Approx. $13 \mathrm{c}$ \\
\hline $\begin{array}{l}\text { Add. 14,735, } \\
\text { foll. 51-71 }\end{array}$ & 3.1148 & DCCCCLXIX & Approx. 12c-13c \\
\hline $\begin{array}{l}\text { Add. 14,735, } \\
\text { foll. } 72-173\end{array}$ & $3.1120-1122$ & DCCCCLVIII & Approx. 12c \\
\hline $\begin{array}{l}\text { Add. 14,736, } \\
\text { fol. } 12\end{array}$ & 3.1153 & DCCCCLXXXV & Approx. 14c \\
\hline $\begin{array}{l}\text { Add. 14,736, } \\
\text { fol. } 3\end{array}$ & 2.1026 & DCCCCIV & Approx. 13c \\
\hline $\begin{array}{l}\text { Add. 14,736, } \\
\text { fol. } 33\end{array}$ & 1.210 & CCLXVIII & Approx. 13c \\
\hline $\begin{array}{l}\text { Add. 14,736, } \\
\text { fol. } 34\end{array}$ & 1.210 & CCLXIX & Approx. $13 \mathrm{c}$ \\
\hline $\begin{array}{l}\text { Add. 14,736, } \\
\text { fol. } 48\end{array}$ & 1.396 & DXIX & Approx. 13c \\
\hline $\begin{array}{l}\text { Add. 14,736, } \\
\text { fol. } 58\end{array}$ & 1.378 & CCCCLXXXIII & Approx. 15c \\
\hline $\begin{array}{l}\text { Add. 14,736, } \\
\text { fol. } 59\end{array}$ & 1.378 & CCCCLXXXIV & Approx. $15 \mathrm{c}$ \\
\hline $\begin{array}{l}\text { Add. 14,736, } \\
\text { fol. } 60\end{array}$ & 1.391 & DXII & Approx. 15c \\
\hline
\end{tabular}




\begin{tabular}{|c|c|c|c|}
\hline Shelfmark & $\begin{array}{l}\text { Catalog pp. } \\
\text { (volume. } \\
\text { pages) }\end{array}$ & $\begin{array}{l}\text { Wright's } \\
\text { Numeration }\end{array}$ & Wright's Dating \\
\hline $\begin{array}{l}\text { Add. 14,736, } \\
\text { fol. } 66\end{array}$ & 1.316 & CCCCII & Approx. 16c \\
\hline $\begin{array}{l}\text { Add. 14,736, } \\
\text { foll. 1, } 2\end{array}$ & 2.591 & DCCV & Approx. 13c-14c \\
\hline $\begin{array}{l}\text { Add. 14,736, } \\
\text { foll. } 10,11\end{array}$ & 2.517 & DCLXIX & Approx. $12 \mathrm{c}-13 \mathrm{c}$ \\
\hline $\begin{array}{l}\text { Add. 14,736, } \\
\text { foll. 13-21 }\end{array}$ & 1.174 & CCXXXI & Approx. 13c \\
\hline $\begin{array}{l}\text { Add. 14,736, } \\
\text { foll. } 22-32\end{array}$ & 2.1022 & DCCCLXXVIII & Approx. 13c \\
\hline $\begin{array}{l}\text { Add. 14,736, } \\
\text { foll. 35-47 }\end{array}$ & 1.303 & CCCLXX & Approx. 12c-13c \\
\hline $\begin{array}{l}\text { Add. 14,736, } \\
\text { foll. 4-9 }\end{array}$ & 2.416 & DXLV & Approx. 13c \\
\hline $\begin{array}{l}\text { Add. 14,736, } \\
\text { foll. 49-52 }\end{array}$ & 1.390 & DX & 1483 \\
\hline $\begin{array}{l}\text { Add. 14,736, } \\
\text { foll. 53-55 }\end{array}$ & 1.296 & CCCLVII & Approx. 13c \\
\hline $\begin{array}{l}\text { Add. 14,736, } \\
\text { foll. } 56,57\end{array}$ & 1.378 & CCCCLXXXII & Approx. 15c \\
\hline $\begin{array}{l}\text { Add. 14,736, } \\
\text { foll. 61-63 }\end{array}$ & 1.301 & CCCLXV & Approx. 15c \\
\hline $\begin{array}{l}\text { Add. } 14,736 \text {, } \\
\text { foll. } 64,65\end{array}$ & $1.315-316$ & CCCXCIX & 1492 \\
\hline $\begin{array}{l}\text { Add. 14,737, } \\
\text { fol. } 50\end{array}$ & 1.212 & CCLXXVII & Approx. 14c \\
\hline $\begin{array}{l}\text { Add. 14,737, } \\
\text { fol. } 92\end{array}$ & 2.517 & DCLXX & Approx. 13c-14c \\
\hline $\begin{array}{l}\text { Add. 14,737, } \\
\text { fol. } 98\end{array}$ & 3.1199 & MXXXI & 1332 \\
\hline $\begin{array}{l}\text { Add. 14,737, } \\
\text { fol. } 99\end{array}$ & $3.1199-1200$ & MXXXIII & 1493 \\
\hline Add. 14,737, & 1.175 & CCXXXV & Approx. 15c \\
\hline
\end{tabular}




\begin{tabular}{|c|c|c|c|}
\hline Shelfmark & $\begin{array}{l}\text { Catalog pp. } \\
\text { (volume. } \\
\text { pages) }\end{array}$ & $\begin{array}{l}\text { Wright's } \\
\text { Numeration }\end{array}$ & Wright's Dating \\
\hline \multicolumn{4}{|l|}{ foll. $1-10$} \\
\hline $\begin{array}{l}\text { Add. 14,737, } \\
\text { foll. 11-17 }\end{array}$ & 1.175 & CCXXXIV & Approx. 14c \\
\hline $\begin{array}{l}\text { Add. 14,737, } \\
\text { foll. 18-34 }\end{array}$ & 1.210 & CCLXX & Approx. 13c \\
\hline $\begin{array}{l}\text { Add. 14,737, } \\
\text { foll. } 35-49\end{array}$ & 1.212 & CCLXXVI & Approx. 14c \\
\hline $\begin{array}{l}\text { Add. 14,737, } \\
\text { foll. 51, } 52\end{array}$ & 1.207 & CCLXII & Approx. 12c-13c \\
\hline $\begin{array}{l}\text { Add. 14,737, } \\
\text { foll. } 53,54\end{array}$ & 1.213 & CCLXXX & Approx. 15c \\
\hline $\begin{array}{l}\text { Add. 14,737, } \\
\text { foll. } 55-58\end{array}$ & 1.314 & CCCXCVIII & Approx. 13c-14c \\
\hline $\begin{array}{l}\text { Add. 14,737, } \\
\text { foll. 59-64 }\end{array}$ & 1.377 & CCCCLXXIV & Approx. 13c \\
\hline $\begin{array}{l}\text { Add. } 14,737 \text {, } \\
\text { foll. } 65-70\end{array}$ & 1.297 & CCCLIX & Approx. 12c-13c \\
\hline $\begin{array}{l}\text { Add. 14,737, } \\
\text { foll. } 71,72\end{array}$ & 1.276 & CCCXXXI & Approx. 12c \\
\hline $\begin{array}{l}\text { Add. 14,737, } \\
\text { foll. } 73-76\end{array}$ & 1.377 & CCCCLXXV & Approx. 13c \\
\hline $\begin{array}{l}\text { Add. 14,737, } \\
\text { foll. } 77-84\end{array}$ & $1.274-275$ & CCCXXIX & 1175 \\
\hline $\begin{array}{l}\text { Add. 14,737, } \\
\text { foll. } 85,86\end{array}$ & 3.1146 & DCCCCLXV & Approx. 13c \\
\hline $\begin{array}{l}\text { Add. 14,737, } \\
\text { foll. 87-91 }\end{array}$ & 2.1026 & DCCCCIII & Approx. 13c \\
\hline $\begin{array}{l}\text { Add. 14,738, } \\
\text { fol. } 1\end{array}$ & 1.7 & VIII & Approx. 14c \\
\hline $\begin{array}{l}\text { Add. 14,738, } \\
\text { fol. } 10\end{array}$ & 1.174 & CCXXXII & Approx. 13c \\
\hline $\begin{array}{l}\text { Add. 14,738, } \\
\text { fol. } 105\end{array}$ & 3.1153 & $\begin{array}{l}\text { DCCCCLXXXV } \\
\text { I }\end{array}$ & Approx. 13c \\
\hline
\end{tabular}




\begin{tabular}{|l|l|l|l|}
\hline Shelfmark & $\begin{array}{l}\text { Catalog pp. } \\
\text { (volume. } \\
\text { pages) }\end{array}$ & $\begin{array}{l}\text { Wright's } \\
\text { Numeration }\end{array}$ & Wright's Dating \\
\hline $\begin{array}{l}\text { Add. 14,738, } \\
\text { fol. 116 }\end{array}$ & 2.1026 & DCCCCI & Approx. 12c \\
\hline $\begin{array}{l}\text { Add. 14,738, } \\
\text { fol. 119 }\end{array}$ & 2.1026 & DCCCXCIX & Approx. 11c-12c \\
\hline $\begin{array}{l}\text { Add. 14,738, } \\
\text { fol. 120 }\end{array}$ & 3.1199 & MXXXII & 1413 \\
\hline $\begin{array}{l}\text { Add. 14,738, } \\
\text { fol. 84 }\end{array}$ & 1.377 & CCCCLXXVIII & Approx. 13c-14c \\
\hline $\begin{array}{l}\text { Add. 14,738, } \\
\text { foll. 106, 107 }\end{array}$ & 3.1149 & DCCCCLXX & Approx. 12c-13c \\
\hline $\begin{array}{l}\text { Add. 14,738, } \\
\text { foll. 108,109 }\end{array}$ & 2.1026 & DCCCCII & Approx. 12c-13c \\
\hline $\begin{array}{l}\text { Add. 14,738, } \\
\text { foll. 11-22 }\end{array}$ & 1.212 & CCLXXV & Approx. 14c \\
\hline $\begin{array}{l}\text { Add. 14,738, } \\
\text { foll. 110-113 }\end{array}$ & 2.1026 & DCCCC & Approx. 12c \\
\hline $\begin{array}{l}\text { Add. 14,738, } \\
\text { foll. 114, 115 }\end{array}$ & 3.1164 & DCCCCXCI & Approx. 11c \\
\hline $\begin{array}{l}\text { Add. 14,738, } \\
\text { foll. 117, 118 }\end{array}$ & 2.516 & DCLXV & Approx. 11c \\
\hline $\begin{array}{l}\text { Add. 14,738, } \\
\text { foll. 2-5 }\end{array}$ & 1.136 & CXCII & Approx. 13c \\
\hline $\begin{array}{l}\text { Add. 14,738, } \\
\text { foll. 23-34 }\end{array}$ & 1.210 & CCLXXI & Approx. 13c \\
\hline $\begin{array}{l}\text { Add. 14,738, } \\
\text { foll. 35, 36 }\end{array}$ & 1.213 & CCLXXIX & Approx. 14c \\
\hline $\begin{array}{l}\text { Add. 14,738, } \\
\text { foll. 37-60 }\end{array}$ & 1.279 & CCCXXXVI & Approx. 13c \\
\hline $\begin{array}{l}\text { Add. 14,738, } \\
\text { foll. 6, 7 }\end{array}$ & 1.83 & CXXX & Approx. 13c \\
\hline $\begin{array}{l}\text { Add. 14,738, } \\
\text { foll. 61-63 }\end{array}$ & 1.377 & Approx. 13c-14c \\
\hline Add. 14,738, & 1.381 & Approx. 13c \\
\hline
\end{tabular}




\begin{tabular}{|c|c|c|c|}
\hline Shelfmark & $\begin{array}{l}\text { Catalog pp. } \\
\text { (volume. } \\
\text { pages) }\end{array}$ & $\begin{array}{l}\text { Wright's } \\
\text { Numeration }\end{array}$ & Wright's Dating \\
\hline \multicolumn{4}{|l|}{ foll. $64-76$} \\
\hline $\begin{array}{l}\text { Add. } 14,738, \\
\text { foll. } 77,78\end{array}$ & 1.316 & $\mathrm{CCCCV}$ & Approx. 13c \\
\hline $\begin{array}{l}\text { Add. 14,738, } \\
\text { foll. } 8,9\end{array}$ & $1.95-96$ & CLII & Approx. 13c-14c \\
\hline $\begin{array}{l}\text { Add. 14,738, } \\
\text { foll. } 82,83\end{array}$ & 1.377 & CCCCLXXX & Approx. 14c \\
\hline $\begin{array}{l}\text { Add. 14,738, } \\
\text { foll. } 85-89\end{array}$ & 2.890 & DCCCXLIX & Approx. 13c-14c \\
\hline $\begin{array}{l}\text { Add. 14,738, } \\
\text { foll. 90-104 }\end{array}$ & 3.1150 & DCCCCLXXV & Approx. 13c \\
\hline $\begin{array}{l}\text { Add. 14,739, } \\
\text { fol. } 23\end{array}$ & 1.316 & CCCC & 1483 \\
\hline $\begin{array}{l}\text { Add. 14,739, } \\
\text { fol. } 24\end{array}$ & $3.1198-1199$ & MXXIX & Approx. 13c \\
\hline $\begin{array}{l}\text { Add. 14,739, } \\
\text { foll. 1-6 }\end{array}$ & $2.866-867$ & DCCCXXXV & Approx. 12c \\
\hline $\begin{array}{l}\text { Add. 14,739, } \\
\text { foll. 12-14 }\end{array}$ & 2.516 & DCLXIII & Approx. 12c \\
\hline $\begin{array}{l}\text { Add. 14,739, } \\
\text { foll. 15-18 }\end{array}$ & 1.280 & CCCXXXVII & Approx. 13c \\
\hline $\begin{array}{l}\text { Add. 14,739, } \\
\text { foll. 19-22 }\end{array}$ & 1.178 & CCXLII & Approx. 13c-14c \\
\hline $\begin{array}{l}\text { Add. 14,739, } \\
\text { foll. } 7-11\end{array}$ & $2.516-517$ & DCLXVI & Approx. 11c-12c \\
\hline Add. 15,443 & $1.139-141$ & CCIII & 1826 \\
\hline Add. 17,102 & $1.11-12$ & XVII & 599 \\
\hline Add. 17,103 & $1.32-33$ & LII & Approx. 8c \\
\hline Add. 17,104 & $1.16-17$ & $\mathrm{XXV}$ & Approx. 6c \\
\hline Add. 17,105 & $1.22-23$ & XXXVI & Approx. 6c \\
\hline Add. 17,106, & 1.23 & XXXVII & Approx. 6c-7c \\
\hline
\end{tabular}




\begin{tabular}{|c|c|c|c|}
\hline Shelfmark & $\begin{array}{l}\text { Catalog pp. } \\
\text { (volume. } \\
\text { pages) }\end{array}$ & $\begin{array}{l}\text { Wright's } \\
\text { Numeration }\end{array}$ & Wright's Dating \\
\hline \multicolumn{4}{|l|}{ foll. 1-73 } \\
\hline $\begin{array}{l}\text { Add. 17,106, } \\
\text { foll. 74-87 }\end{array}$ & 1.28 & XLVII & Approx. 7c \\
\hline Add. 17,107 & $1.23-24$ & XXXVIII & 541 \\
\hline Add. 17,108 & 1.18 & XXIX & Approx. 6c \\
\hline Add. 17,109 & $1.120-122$ & CLXX & 873-874 \\
\hline Add. 17,110 & 1.116-119 & CLXVIII & 600 (at latest) \\
\hline Add. 17,111 & $1.125-126$ & CLXXVI & 927 \\
\hline Add. 17,112 & 1.127 & CLXXIX & Approx. 10c-11c \\
\hline Add. 17,113 & $1.48-49$ & LXXII & Approx. 6c-7c \\
\hline Add. 17,114 & $1.45-46$ & LXVIII & Approx. 6c-7c \\
\hline Add. 17,115 & 1.66 & XCVI & Approx. 6c \\
\hline Add. 17,116 & 1.65 & XCIII & Approx. 6c \\
\hline Add. 17,117 & 1.64 & XCI & $\begin{array}{ll}\text { Approx. } & 5 c-6 c \\
\text { (early) } & \end{array}$ \\
\hline Add. 17,118 & $1.57-58$ & LXXXI & Approx. 8c-9c \\
\hline Add. 17,119 & $1.71-72$ & CXII & Approx. 6c-7c \\
\hline Add. 17,120 & 1.80 & CXXVI & Approx. 6c \\
\hline Add. 17,121 & $1.80-81$ & CXXVII & Approx. 6c \\
\hline Add. 17,122 & $1.87-88$ & CXXXVII & Approx. 6c \\
\hline Add. 17,123 & 1.93 & CXLV & Approx. 9c-10c \\
\hline Add. 17,124 & $1.42-44$ & LXV & 1234 \\
\hline Add. 17,125 & $1.123-125$ & CLXXV & Approx. 9c-10c \\
\hline Add. 17,126 & 2.526 & DCLXXIV & 511 \\
\hline Add. 17,127 & $2.1020-1021$ & DCCCLXXV & 1088 \\
\hline Add. 17,128 & $1.226-227$ & CCXC & Approx. 10c-11c \\
\hline Add. 17,129 & 1.383 & CCCCXCIV & Approx. 7c-8c \\
\hline Add. 17,130 & $1.392-393$ & DXIII & 877 \\
\hline
\end{tabular}




\begin{tabular}{|c|c|c|c|}
\hline Shelfmark & $\begin{array}{l}\text { Catalog pp. } \\
\text { (volume. } \\
\text { pages) }\end{array}$ & $\begin{array}{l}\text { Wright's } \\
\text { Numeration }\end{array}$ & Wright's Dating \\
\hline Add. 17,131 & 1.395 & DXVI & Approx. 12c \\
\hline Add. 17,132 & 1.371 & CCCCLXVIII & Approx. 12c-13c \\
\hline Add. 17,133 & 1.324 & CCCCXII & Approx. 11c \\
\hline Add. 17,134 & $1.330-339$ & CCCCXXI & 675 \\
\hline Add. 17,135 & $1.293-294$ & CCCL & Approx. 10c \\
\hline Add. 17,136 & $1.343-345$ & CCCCXXVII & Approx. 10c-11c \\
\hline Add. 17,137 & $1.369-370$ & CCCCLXV & Approx. varied \\
\hline Add. 17,138 & 1.295 & CCCLIV & Approx. 11c \\
\hline Add. 17,139 & 1.343 & CCCCXXVI & Approx. 10c-11c \\
\hline Add. 17,140 & $1.345-346$ & CCCCXXIX & Approx. 11c \\
\hline Add. 17,141 & $1.359-363$ & CCCCL & Approx. 8c-9c \\
\hline Add. 17,142 & 3.1042 & DCCCCXVII & Approx. 6c \\
\hline Add. 17,143 & 2.416 & DXLVI & Approx. 5c \\
\hline Add. 17,144 & $2.652-654$ & DCCXXXII & Approx. 6c \\
\hline Add. 17,145 & $2.418-419$ & DXLVIII & Approx. 8c-9c \\
\hline Add. 17,146 & $2.437-438$ & DLX & Approx. 6c-7c \\
\hline Add. 17,147 & $2.438-440$ & DLXI & Approx. 8c-9c \\
\hline Add. 17,148 & $2.717-718$ & DCCLVI & Approx. 7c \\
\hline Add. 17,149 & $2.668-669$ & DCCXXXIX & Approx. 6c \\
\hline Add. 17,150 & $2.492-493$ & DCXXII & Approx. 7c-8c \\
\hline Add. 17,151 & $2.490-491$ & DCXIX & Approx. 7c \\
\hline Add. 17,152 & $2.477-478$ & DXCVI & 594 \\
\hline Add. 17,153 & 2.531 & DCLXXIX & Approx. 6c \\
\hline Add. 17,154 & $2.557-558$ & DCXCI & Approx. 7c \\
\hline Add. 17,155 & $2.507-508$ & DCXXXIX & Approx. 6c-7c \\
\hline Add. 17,156 & $3.1187-1188$ & MV & Approx. 8c \\
\hline Add. 17,156, & $3.1162-1163$ & DCCCCLXXXI & Approx. 9c \\
\hline
\end{tabular}




\begin{tabular}{|l|l|l|l|}
\hline Shelfmark & $\begin{array}{l}\text { Catalog pp. } \\
\text { (volume. } \\
\text { pages) }\end{array}$ & $\begin{array}{l}\text { Wright's } \\
\text { Numeration }\end{array}$ & Wright's Dating \\
\hline foll. 1-12 & & X & \\
\hline $\begin{array}{l}\text { Add. 17,156, } \\
\text { foll. 16-31 }\end{array}$ & 1.302 & CCCLXVII & Approx. 10c \\
\hline Add. 17,157 & $2.504-505$ & DCXXXVI & 565 \\
\hline $\begin{array}{l}\text { Add. 17,158, } \\
\text { fol. 57 }\end{array}$ & 2.416 & DXLIV & Approx. 8c \\
\hline $\begin{array}{l}\text { Add. 17,158, } \\
\text { foll. 1-48 }\end{array}$ & $2.681-683$ & DCCXLVI & Approx. 6c-7c \\
\hline $\begin{array}{l}\text { Add. 17,158, } \\
\text { foll. 49-56 }\end{array}$ & 2.513 & DCLI & Approx. 8c \\
\hline $\begin{array}{l}\text { Add. 17,159, } \\
\text { foll. 1-63 }\end{array}$ & 2.511 & DCXLV & Approx. 7c \\
\hline $\begin{array}{l}\text { Add. 17,159, } \\
\text { foll. 64-93 }\end{array}$ & $2.511-512$ & DCXLVI & Approx. 7c \\
\hline $\begin{array}{l}\text { Add. 17,160, } \\
\text { fol. 27 }\end{array}$ & 2.1025 & DCCCLXXXIX & Approx. 7c \\
\hline $\begin{array}{l}\text { Add. 17,160, } \\
\text { fol. 28 }\end{array}$ & 2.1024 & DCCCLXXXIII & Approx. 6c-7c \\
\hline $\begin{array}{l}\text { Add. 17,160, } \\
\text { foll. 1-18 }\end{array}$ & $2.512-513$ & DCL & Approx. 7c \\
\hline $\begin{array}{l}\text { Add. 17,160, } \\
\text { foll. 19-23 }\end{array}$ & $2.481-482$ & DCIV & Approx. 6c-7c \\
\hline $\begin{array}{l}\text { Add. 17,160, } \\
\text { foll. 24-26 }\end{array}$ & 2.1018 & DCCCLXIX & Approx. 8c \\
\hline $\begin{array}{l}\text { Add. 17,160, } \\
\text { foll. 29, 30 }\end{array}$ & $1.235-236$ & CCCIII & 789 \\
\hline Add. 17,161 & $2.505-506$ & DCXXXVII & Approx. 6c \\
\hline $\begin{array}{l}\text { Add. 17,162, } \\
\text { foll. 1-14 }\end{array}$ & $1.111-112$ & CLXIV & Approx. 10c-11c \\
\hline $\begin{array}{l}\text { Add. 17,162, } \\
\text { foll. 15-27 }\end{array}$ & $2.513-514$ & DCLIV & Approx. 9c \\
\hline Add. 17,163, & $2.524-526$ & DCLXXIII & Approx. 7c \\
\hline
\end{tabular}




\begin{tabular}{|c|c|c|c|}
\hline Shelfmark & $\begin{array}{l}\text { Catalog pp. } \\
\text { (volume. } \\
\text { pages) }\end{array}$ & $\begin{array}{l}\text { Wright's } \\
\text { Numeration }\end{array}$ & Wright's Dating \\
\hline \multicolumn{4}{|l|}{ foll. 1-48 } \\
\hline $\begin{array}{l}\text { Add. 17,163, } \\
\text { foll. 49-53 }\end{array}$ & 2.1018 & DCCCLXX & Approx. 9c \\
\hline Add. 17,164 & $2.680-681$ & DCCXLV & Approx. 6c-7c \\
\hline Add. 17,165 & $2.654-655$ & DCCXXXIII & Approx. 6c \\
\hline Add. 17,166 & $2.658-660$ & DCCXXXVII & Approx. 6c \\
\hline Add. 17,167 & $2.676-678$ & DCCXLIII & Approx. 6c-7c \\
\hline $\begin{array}{l}\text { Add. 17,168, } \\
\text { fol. 1-113 }\end{array}$ & $2.782-784$ & DCCXCII & Approx. 9c \\
\hline $\begin{array}{l}\text { Add. 17,168, } \\
\text { foll. 114-153 }\end{array}$ & $2.777-778$ & DCCLXXXVIII & Approx. 9c \\
\hline $\begin{array}{l}\text { Add. 17,168, } \\
\text { foll. 154-184 }\end{array}$ & 2.800 & DCCXCIX & Approx. 9c \\
\hline Add. 17,169 & $2.450-454$ & DLXXII & 581 \\
\hline Add. 17,170 & $2.454-458$ & DLXXIII & $774-5$ \\
\hline $\begin{array}{l}\text { Add. 17,171, } \\
\text { foll. 1-16 }\end{array}$ & 2.657 & DCCXXXV & Approx. 6c \\
\hline $\begin{array}{l}\text { Add. 17,171, } \\
\text { foll. 17-23 }\end{array}$ & 2.513 & DCLII & Approx. 8c \\
\hline Add. 17,172 & $2.759-762$ & DCCLXXX & $819-830$ \\
\hline Add. 17,173 & $2.726-729$ & DCCLXII & Approx. 7c \\
\hline Add. 17,174 & $3.1074-1076$ & DCCCCXXVIII & 929 \\
\hline Add. 17,175 & 3.1080 & DCCCCXXXII & Approx. 10c \\
\hline Add. 17,176 & $3.1072-1073$ & DCCCCXXIV & 532 \\
\hline Add. 17,177 & $3.1073-1074$ & DCCCCXXV & Approx. 6c \\
\hline Add. 17,178 & $2.855-857$ & DCCCXXVIII & Approx. 11c-12c \\
\hline Add. 17,179 & $2.857-858$ & DCCCXXIX & Approx. 11c-12c \\
\hline Add. 17,180 & $2.851-853$ & DCCCXXVI & Approx. 11c \\
\hline Add. 17,181 & $2.661-668$ & DCCXXXVIII & Approx. 6c \\
\hline
\end{tabular}




\begin{tabular}{|l|l|l|l|}
\hline Shelfmark & $\begin{array}{l}\text { Catalog pp. } \\
\text { (volume. } \\
\text { pages) }\end{array}$ & $\begin{array}{l}\text { Wright's } \\
\text { Numeration }\end{array}$ & Wright's Dating \\
\hline $\begin{array}{l}\text { Add. 17,182, } \\
\text { foll. 1-99 }\end{array}$ & $2.403-404$ & DXXIX & 474 \\
\hline $\begin{array}{l}\text { Add. 17,182, } \\
\text { foll. 100-175 }\end{array}$ & $2.404-405$ & DXXX & 512 \\
\hline Add. 17,183 & $2.819-823$ & DCCCXII & Approx. 10c \\
\hline Add. 17,184 & $2.509-510$ & DCXLI & Approx. 6c-7c \\
\hline Add. 17,185 & $2.838-840$ & DCCCXXII & Approx. 10c-11c \\
\hline Add. 17,186 & $2.420-421$ & DLI & Approx. 6c \\
\hline Add. 17,187 & 2.605 & DCCVIII & Approx. 10c \\
\hline Add. 17,188 & $2.620-622$ & DCCXXI & Approx. 10c-11c \\
\hline $\begin{array}{l}\text { Add. 17,189, } \\
\text { foll. 1-16 }\end{array}$ & 2.407 & DXXXIV & Approx. 5c-6c \\
\hline $\begin{array}{l}\text { Add. 17,189, } \\
\text { foll. 17-21 }\end{array}$ & 2.1016 & DCCCLXV & Approx. 6c \\
\hline Add. 17,190 & $1.243-244$ & CCCVII & 893 \\
\hline Add. 17,191 & $2.1008-1015$ & DCCCLXIV & Approx. 9c-10c \\
\hline Add. 17,192 & $2.778-781$ & DCCLXXXIX & Approx. 9c \\
\hline Add. 17,193 & $2.989-1002$ & DCCCLXI & 874 \\
\hline Add. 17,194 & $2.1002-1003$ & DCCCLXII & 886 \\
\hline Add. 17,195 & $2.914-915$ & DCCCLIV & Approx. 10c \\
\hline Add. 17,196 & $2.776-777$ & DCCLXXXVII & Approx. 6c \\
\hline $\begin{array}{l}\text { Add. 17,197, } \\
\text { foll. 1-25 }\end{array}$ & $2.440-441$ & DLXII & Approx. 9c-10c \\
\hline $\begin{array}{l}\text { Add. 17,197, } \\
\text { foll. 26-46 }\end{array}$ & $2.606-608$ & DCCXI & Approx. 9c-10c \\
\hline Add. 17,198 & 2.503 & DCXXXII & Approx. 7c \\
\hline Add. 17,199 & $2.612-613$ & DCCXV & Approx. 8c \\
\hline Add. 17,200 & $2.554-555$ & DCLXXXIX & Approx. 7c \\
\hline $\begin{array}{l}\text { Add. 17,201, } \\
\text { foll. 1-15 }\end{array}$ & 2.690 & DCCXLIX & Approx. 6c-7c \\
\hline
\end{tabular}




\begin{tabular}{|c|c|c|c|}
\hline Shelfmark & $\begin{array}{l}\text { Catalog pp. } \\
\text { (volume. } \\
\text { pages) }\end{array}$ & $\begin{array}{l}\text { Wright's } \\
\text { Numeration }\end{array}$ & Wright's Dating \\
\hline $\begin{array}{l}\text { Add. 17,201, } \\
\text { foll. 16-21 }\end{array}$ & 2.458 & DLXXIV & Approx. 8c \\
\hline $\begin{array}{l}\text { Add. 17,201, } \\
\text { foll. 22-25 }\end{array}$ & 3.1149 & DCCCCLXXIII & Approx. $7 c-8 c$ \\
\hline $\begin{array}{l}\text { Add. 17,201, } \\
\text { foll. 26-32 }\end{array}$ & 2.465 & DLXXXI & Approx. 9c-10c \\
\hline Add. 17,202 & $3.1046-1061$ & DCCCCXIX & $\begin{array}{l}\text { Approx. 6c (late)- } \\
\text { 7c(early) }\end{array}$ \\
\hline Add. 17,203 & 3.1151 & DCCCCLXXX & Approx. 11c \\
\hline Add. 17,204 & 3.1081 & DCCCCXXXIV & Approx. 5c \\
\hline Add. 17,205 & 3.1087 & $\begin{array}{l}\text { DCCCCXXXVII } \\
\text { I }\end{array}$ & Approx. 6c \\
\hline Add. 17,206 & $2.859-860$ & DCCCXXXI & Approx. 11c-12c \\
\hline Add. 17,207 & $1.365-366$ & CCCCLIII & Approx. 8c-9c \\
\hline Add. 17,208 & $2.613-617$ & DCCXVII & Approx. 9c \\
\hline Add. 17,209 & $3.1184-1186$ & MIII & Approx. 9c \\
\hline $\begin{array}{l}\text { Add. 17,210- } \\
211\end{array}$ & $2.548-550$ & DCLXXXVII & Approx. 5c \\
\hline Add. 17,212 & $2.479-480$ & DXCVIII & Approx. 9c-10c \\
\hline $\begin{array}{l}\text { Add. 17,213, } \\
\text { fol. } 13\end{array}$ & 2.515 & DCLIX & Approx. 10c \\
\hline $\begin{array}{l}\text { Add. 17,213, } \\
\text { fol. } 14\end{array}$ & $2.515-516$ & DCLXI & Approx. 11c \\
\hline $\begin{array}{l}\text { Add. 17,213, } \\
\text { fol. } 15\end{array}$ & 2.516 & DCLXII & Approx. 11c \\
\hline $\begin{array}{l}\text { Add. 17,213, } \\
\text { fol. } 18\end{array}$ & 2.516 & DCLXIV & Approx. 10c \\
\hline $\begin{array}{l}\text { Add. 17,213, } \\
\text { fol. } 19\end{array}$ & 3.1150 & DCCCCLXXVII & Approx. 10c-11c \\
\hline $\begin{array}{l}\text { Add. 17,213, } \\
\text { fol. } 20\end{array}$ & $1.295-296$ & CCCLV & 1101 \\
\hline
\end{tabular}




\begin{tabular}{|l|l|l|l|}
\hline Shelfmark & $\begin{array}{l}\text { Catalog pp. } \\
\text { (volume. } \\
\text { pages) }\end{array}$ & $\begin{array}{l}\text { Wright's } \\
\text { Numeration }\end{array}$ & Wright's Dating \\
\hline $\begin{array}{l}\text { Add. 17,213, } \\
\text { fol. 3 }\end{array}$ & 1.28 & XLVI & Approx. 9c \\
\hline $\begin{array}{l}\text { Add. 17,213, } \\
\text { fol. 40 }\end{array}$ & 1.176 & CCXXXVIII & Approx. 13c \\
\hline $\begin{array}{l}\text { Add. 17,213, } \\
\text { fol. 41 }\end{array}$ & 3.1198 & MXXVII & 1209 \\
\hline $\begin{array}{l}\text { Add. 17,213, } \\
\text { fol. 42 }\end{array}$ & 3.1197 & MXXII & Approx. 10c \\
\hline $\begin{array}{l}\text { Add. 17,213, } \\
\text { fol. 43 }\end{array}$ & 3.1194 & MX & Approx. 6c-7c \\
\hline $\begin{array}{l}\text { Add. 17,213, } \\
\text { foll. 1,2 }\end{array}$ & 1.37 & LVII & Approx. 9c \\
\hline $\begin{array}{l}\text { Add. 17,213, } \\
\text { foll. 11,12 }\end{array}$ & 2.515 & DCLVII & Approx. 9c-10c \\
\hline $\begin{array}{l}\text { Add. 17,213, } \\
\text { foll. 16,17 }\end{array}$ & 2.515 & DCLX & Approx. 10c \\
\hline $\begin{array}{l}\text { Add. 17,213, } \\
\text { foll. 21-39 }\end{array}$ & 2.781 & DCCXC & Approx. 9c \\
\hline $\begin{array}{l}\text { Add. 17,213, } \\
\text { foll. 4,5 }\end{array}$ & 2.401 & DXXVII & Approx. 6c \\
\hline $\begin{array}{l}\text { Add. 17,213, } \\
\text { foll. 41, 42 }\end{array}$ & 1.367 & CCCCLIX & Approx. 11c \\
\hline $\begin{array}{l}\text { Add. 17,213, } \\
\text { foll. 6, 7 }\end{array}$ & 2.1025 & DCCCXCI & Approx. 8c-9c \\
\hline $\begin{array}{l}\text { Add. 17,213, } \\
\text { foll. 8-10 }\end{array}$ & 2.752 & DCCLXXVI & Approx. 8c-9c \\
\hline Add. 17,214 & $2.915-917$ & DCCCLV & Approx. 7c \\
\hline $\begin{array}{l}\text { Add. 17,215, } \\
\text { fol. 34 }\end{array}$ & $2.503-504$ & DCXXXIV & Approx. 8c-9c \\
\hline $\begin{array}{l}\text { Add. 17,215, } \\
\text { fol. 48 }\end{array}$ & $3.1196-1197$ \\
Add. 17,215, & 3.1198 & MXVIII & Approx. 9c-10c \\
\hline
\end{tabular}




\begin{tabular}{|c|c|c|c|}
\hline Shelfmark & $\begin{array}{l}\text { Catalog pp. } \\
\text { (volume. } \\
\text { pages) }\end{array}$ & $\begin{array}{l}\text { Wright's } \\
\text { Numeration }\end{array}$ & Wright's Dating \\
\hline $\begin{array}{l}\text { Add. 17,215, } \\
\text { fol. } 50\end{array}$ & 3.1200 & MXXXVI & $\begin{array}{l}\text { Approx. } \quad \text { (not } \\
\text { given) }\end{array}$ \\
\hline $\begin{array}{l}\text { Add. 17,215, } \\
\text { foll. 1-4 }\end{array}$ & 2.1018-1019 & DCCCLXXII & Approx. 9c-10c \\
\hline $\begin{array}{l}\text { Add. 17,215, } \\
\text { foll. } 22-25\end{array}$ & 1.388 & DIII & Approx. 10c-11c \\
\hline $\begin{array}{l}\text { Add. 17,215, } \\
\text { foll. } 26,27\end{array}$ & 2.1025 & DCCCXCII & Approx. 8c-9c \\
\hline $\begin{array}{l}\text { Add. 17,215, } \\
\text { foll. } 28,29\end{array}$ & 2.493 & DCXXIII & Approx. 10c \\
\hline $\begin{array}{l}\text { Add. 17,215, } \\
\text { foll. 30-33 }\end{array}$ & 2.1016 & DCCCLXVI & Approx. 7c-8c \\
\hline $\begin{array}{l}\text { Add. 17,215, } \\
\text { foll. 35-43 }\end{array}$ & 2.840 & DCCCXXIII & Approx. 10c-11c \\
\hline $\begin{array}{l}\text { Add. 17,215, } \\
\text { foll. } 44,45\end{array}$ & 2.1026 & DCCCXCVI & Approx. 9c \\
\hline $\begin{array}{l}\text { Add. 17,215, } \\
\text { foll. 46, } 47\end{array}$ & 3.1074 & DCCCCXXVII & Approx. 8c \\
\hline $\begin{array}{l}\text { Add. 17,215, } \\
\text { foll. 5, } 6\end{array}$ & $3.1164-1165$ & DCCCCXCIII & 839 \\
\hline $\begin{array}{l}\text { Add. 17,215, } \\
\text { foll. } 7,8\end{array}$ & 3.1165 & DCCCCXCIV & Approx. 9c \\
\hline $\begin{array}{l}\text { Add. 17,215, } \\
\text { foll. 9-21 }\end{array}$ & 2.810 & DCCCV & Approx. 9c-10c \\
\hline $\begin{array}{l}\text { Add. 17,216, } \\
\text { fol. } 1\end{array}$ & 3.1041 & DCCCCXIV & Approx. 8c-9c \\
\hline $\begin{array}{l}\text { Add. 17,216, } \\
\text { fol. } 43\end{array}$ & 2.1037 & DCCCCX & Approx. 8c-9c \\
\hline $\begin{array}{l}\text { Add. 17,216, } \\
\text { fol. } 44\end{array}$ & 3.1150 & DCCCCLXXVI & Approx. 9c \\
\hline $\begin{array}{l}\text { Add. 17,216, } \\
\text { fol. } 45\end{array}$ & 3.1147 & DCCCCLXVI & Approx. 9c-10c \\
\hline Add. 17,216, & 1.367 & CCCCLXI & Approx. 11c \\
\hline
\end{tabular}




\begin{tabular}{|c|c|c|c|}
\hline Shelfmark & $\begin{array}{l}\text { Catalog pp. } \\
\text { (volume. } \\
\text { pages) }\end{array}$ & $\begin{array}{l}\text { Wright's } \\
\text { Numeration }\end{array}$ & Wright's Dating \\
\hline \multicolumn{4}{|l|}{ fol. 46} \\
\hline $\begin{array}{l}\text { Add. 17,216, } \\
\text { fol. } 47\end{array}$ & $3.1195-1196$ & MXV & 869 \\
\hline $\begin{array}{l}\text { Add. 17,216, } \\
\text { fol. } 48\end{array}$ & 3.1195 & MXIV & 849 \\
\hline $\begin{array}{l}\text { Add. 17,216, } \\
\text { fol. } 49\end{array}$ & 3.1197 & MXX & (932) \\
\hline $\begin{array}{l}\text { Add. 17,216, } \\
\text { fol. } 50\end{array}$ & 3.1194 & MXI & $\begin{array}{l}\text { Approx. 10c/6c- } \\
7 \mathrm{c}\end{array}$ \\
\hline $\begin{array}{l}\text { Add. 17,216, } \\
\text { fol. } 51\end{array}$ & 3.1197 & MXXI & (932) \\
\hline $\begin{array}{l}\text { Add. 17,216, } \\
\text { foll. 15-25 }\end{array}$ & 1.272 & CCCXXVI & Approx. 11c \\
\hline $\begin{array}{l}\text { Add. 17,216, } \\
\text { foll. 2-14 }\end{array}$ & 3.1041 & DCCCCXV & Approx. 8c-9c \\
\hline $\begin{array}{l}\text { Add. 17,216, } \\
\text { foll. } 26,27\end{array}$ & 1.302 & CCCLXVIII & Approx. 10c \\
\hline $\begin{array}{l}\text { Add. 17,216, } \\
\text { foll. 28-31 }\end{array}$ & 1.366 & CCCCLVI & Approx. 9c \\
\hline $\begin{array}{l}\text { Add. 17,216, } \\
\text { foll. } 32,33\end{array}$ & $2.533-534$ & DCLXXXIV & Approx. 13c \\
\hline $\begin{array}{l}\text { Add. 17,216, } \\
\text { foll. 34-42 }\end{array}$ & 1.370 & CCCCLXVI & Approx. 12c \\
\hline $\begin{array}{l}\text { Add. 17,216, } \\
\text { foll. 52, 53 }\end{array}$ & 3.1196 & MXVII & Approx. 9c \\
\hline $\begin{array}{l}\text { Add. 17,217, } \\
\text { fol. } 39\end{array}$ & $2.488-489$ & DCXVI & Approx. 6c-7c \\
\hline $\begin{array}{l}\text { Add. 17,217, } \\
\text { fol. } 40\end{array}$ & 2.1024 & DCCCLXXXVI & Approx. 6c-7c \\
\hline $\begin{array}{l}\text { Add. 17,217, } \\
\text { fol. } 41\end{array}$ & $2.484-485$ & DCX & Approx. 6c-7c \\
\hline $\begin{array}{l}\text { Add. 17,217, } \\
\text { fol. } 42\end{array}$ & 2.1025 & DCCCXC & Approx. 7c-8c \\
\hline
\end{tabular}




\begin{tabular}{|c|c|c|c|}
\hline Shelfmark & $\begin{array}{l}\text { Catalog pp. } \\
\text { (volume. } \\
\text { pages) }\end{array}$ & $\begin{array}{l}\text { Wright's } \\
\text { Numeration }\end{array}$ & Wright's Dating \\
\hline $\begin{array}{l}\text { Add. 17,217, } \\
\text { fol. } 43\end{array}$ & 2.793 & DCCXCVI & Approx. 9c \\
\hline $\begin{array}{l}\text { Add. 17,217, } \\
\text { fol. } 44\end{array}$ & 2.1025 & DCCCXCIII & Approx. 9c \\
\hline $\begin{array}{l}\text { Add. 17,217, } \\
\text { fol. } 45\end{array}$ & 3.1101 & DCCCCXLVII & Approx. 7c-8c \\
\hline $\begin{array}{l}\text { Add. 17,217, } \\
\text { fol. } 48\end{array}$ & $2.1024-1025$ & DCCCLXXXVII & Approx. 6c-7c \\
\hline $\begin{array}{l}\text { Add. 17,217, } \\
\text { fol. } 49\end{array}$ & 2.1025 & $\begin{array}{l}\text { DCCCLXXXVII } \\
\text { I }\end{array}$ & Approx. 7c \\
\hline $\begin{array}{l}\text { Add. 17,217, } \\
\text { fol. } 50\end{array}$ & 2.1024 & DCCCLXXXIV & Approx. 6c-7c \\
\hline $\begin{array}{l}\text { Add. 17,217, } \\
\text { fol. } 51\end{array}$ & 2.1024 & DCCCLXXXV & Approx. 6c-7c \\
\hline $\begin{array}{l}\text { Add. 17,217, } \\
\text { fol. } 52\end{array}$ & 2.1024 & DCCCLXXXI & Approx. 6c \\
\hline $\begin{array}{l}\text { Add. 17,217, } \\
\text { fol. } 53\end{array}$ & $2.1025-1026$ & DCCCXCV & Approx. 9c \\
\hline $\begin{array}{l}\text { Add. 17,217, } \\
\text { fol. } 54\end{array}$ & 1.146 & CCXIX & Approx. 6c-7c \\
\hline $\begin{array}{l}\text { Add. 17,217, } \\
\text { fol. } 59\end{array}$ & 3.1195 & MXIII & Approx. 8c \\
\hline $\begin{array}{l}\text { Add. 17,217, } \\
\text { fol. } 60\end{array}$ & 3.1194 & MIX & Approx. 6c \\
\hline $\begin{array}{l}\text { Add. 17,217, } \\
\text { fol. } 61\end{array}$ & 3.1197 & MXXIII & Approx. 8c \\
\hline $\begin{array}{l}\text { Add. 17,217, } \\
\text { fol. } 63\end{array}$ & 3.1195 & MXII & Approx. illegible \\
\hline $\begin{array}{l}\text { Add. 17,217, } \\
\text { foll. 1-19 }\end{array}$ & 2.613 & DCCXVI & Approx. 9c-10c \\
\hline $\begin{array}{l}\text { Add. 17,217, } \\
\text { foll. 20-32 }\end{array}$ & 2.482 & DCVI & Approx. 9c \\
\hline Add. 17,217, & 2.488 & DCXV & Approx. 8c \\
\hline
\end{tabular}




\begin{tabular}{|l|l|l|l|}
\hline Shelfmark & $\begin{array}{l}\text { Catalog pp. } \\
\text { (volume. } \\
\text { pages) }\end{array}$ & $\begin{array}{l}\text { Wright's } \\
\text { Numeration }\end{array}$ & Wright's Dating \\
\hline foll. 33-36 & & & \\
\hline $\begin{array}{l}\text { Add. 17,217, } \\
\text { foll. 37, 38 }\end{array}$ & $3.1168-1172$ & DCCCCXCVI & Approx. 9c-10c \\
\hline $\begin{array}{l}\text { Add. 17,217, } \\
\text { foll. 46, 47 }\end{array}$ & 2.450 & DLXXI & Approx. 9c-10c \\
\hline $\begin{array}{l}\text { Add. 17,217, } \\
\text { foll. 55, 56 }\end{array}$ & 2.1025 & DCCCXCIV & Approx. 9c \\
\hline $\begin{array}{l}\text { Add. 17,217, } \\
\text { foll. 57, 58 }\end{array}$ & 2.818 & DCCCIX & Approx. 10c \\
\hline $\begin{array}{l}\text { Add. 17,217, } \\
\text { foll. 62 }\end{array}$ & 3.1200 & MXXXIV & $\begin{array}{l}\text { Approx. } \\
\text { given) }\end{array}$ \\
\hline $\begin{array}{l}\text { Add. 17,218, } \\
\text { fol. 3 }\end{array}$ & 1.303 & CCCLXXII & Approx. 9c \\
\hline $\begin{array}{l}\text { Add. 17,218, } \\
\text { fol. 43 }\end{array}$ & 2.415 & DXLIII & Approx. 7c \\
\hline $\begin{array}{l}\text { Add. 17,218, } \\
\text { fol. 47 }\end{array}$ & 1.367 & CCCCLVII & Approx. 10c \\
\hline $\begin{array}{l}\text { Add. 17,218, } \\
\text { fol. 52 }\end{array}$ & 1.384 & CCCCXCVII & Approx. 9c \\
\hline $\begin{array}{l}\text { Add. 17,218, } \\
\text { fol. 55 }\end{array}$ & 1.395 & DXVII & Approx. 12c \\
\hline $\begin{array}{l}\text { Add. 17,218, } \\
\text { fol. 56 }\end{array}$ & 1.316 & CCCCIV & Approx. 11c \\
\hline $\begin{array}{l}\text { Add. 17,218, } \\
\text { fol. 57 }\end{array}$ & 1.274 \\
\hline $\begin{array}{l}\text { Add. 17,218, } \\
\text { fol. 58 }\end{array}$ & 2.1026 & CCCXXVIII & Approx. 11c-12c \\
\hline $\begin{array}{l}\text { Add. 17,218, } \\
\text { fol. 84 }\end{array}$ & 2.1024 & DCCCLXXXII & Approx. 6c \\
\hline $\begin{array}{l}\text { Add. 17,218, } \\
\text { fol. 89 }\end{array}$ & 2.1026 \\
\hline Add. 17,218, \\
fol. 90
\end{tabular}




\begin{tabular}{|c|c|c|c|}
\hline Shelfmark & $\begin{array}{l}\text { Catalog pp. } \\
\text { (volume. } \\
\text { pages) }\end{array}$ & $\begin{array}{l}\text { Wright's } \\
\text { Numeration }\end{array}$ & Wright's Dating \\
\hline $\begin{array}{l}\text { Add. 17,218, } \\
\text { fol. } 97\end{array}$ & 3.1200 & MXXXV & $\begin{array}{l}\text { Approx. } \quad \text { (not } \\
\text { given) }\end{array}$ \\
\hline $\begin{array}{l}\text { Add. 17,218, } \\
\text { foll, } 53,54\end{array}$ & 1.233 & CCXCVII & Approx. 8c \\
\hline $\begin{array}{l}\text { Add. 17,218, } \\
\text { foll. } 23-40\end{array}$ & 1.154 & CCXXIII & Approx. 9c-10c \\
\hline $\begin{array}{l}\text { Add. 17,218, } \\
\text { foll. 4-22 }\end{array}$ & 1.202 & CCLII & Approx. 11c \\
\hline $\begin{array}{l}\text { Add. 17,218, } \\
\text { foll. } 44-46\end{array}$ & 1.365 & CCCCLII & Approx. 8c-9c \\
\hline $\begin{array}{l}\text { Add. } 17,218, \\
\text { foll. } 48,49\end{array}$ & 1.384 & CCCCXCVIII & Approx. 9c-10c \\
\hline $\begin{array}{l}\text { Add. 17,218, } \\
\text { foll. } 50,51\end{array}$ & 1.367 & CCCCLVIII & Approx. 11c \\
\hline $\begin{array}{l}\text { Add. 17,218, } \\
\text { foll. 59-69 }\end{array}$ & 2.514 & DCLV & Approx. 9c \\
\hline $\begin{array}{l}\text { Add. 17,218, } \\
\text { foll. } 70-83\end{array}$ & 2.806 & DCCCII & Approx. 9c \\
\hline $\begin{array}{l}\text { Add. 17,218, } \\
\text { foll. } 85,86\end{array}$ & 2.1018 & DCCCLXXI & Approx. 9c \\
\hline $\begin{array}{l}\text { Add. 17,218, } \\
\text { foll. } 87,88\end{array}$ & $2.1016-1017$ & DCCCLXVII & Approx. 7c-8c \\
\hline $\begin{array}{l}\text { Add. 17,218, } \\
\text { foll. 91-96 }\end{array}$ & 1.177 & CCXL & Approx. 12c \\
\hline Add. 17,219 & $1.134-136$ & CXCI & Approx. 13c \\
\hline Add. 17,220 & $1.133-134$ & $\mathrm{CXC}$ & Approx. 13c \\
\hline Add. 17,221 & 1.145 & CCXVIII & Approx. 14c-15c \\
\hline Add. 17,222 & 1.143 & CCX & Approx. 13c \\
\hline Add. 17,223 & 1.137 & CXCVII & Approx. 14c \\
\hline $\begin{array}{l}\text { Add. 17,224, } \\
\text { fol. } 18\end{array}$ & 1.298 & CCCLXII & Approx. 13c \\
\hline
\end{tabular}




\begin{tabular}{|l|l|l|l|}
\hline Shelfmark & $\begin{array}{l}\text { Catalog pp. } \\
\text { (volume. } \\
\text { pages) }\end{array}$ & $\begin{array}{l}\text { Wright's } \\
\text { Numeration }\end{array}$ & Wright's Dating \\
\hline $\begin{array}{l}\text { Add. 17,224, } \\
\text { fol. 36 }\end{array}$ & 3.1198 & MXXVIII & Approx. 13c \\
\hline $\begin{array}{l}\text { Add. 17,224, } \\
\text { fol. 66 }\end{array}$ & 1.71 & CXI & Approx. 13c \\
\hline $\begin{array}{l}\text { Add. 17,224, } \\
\text { fol. 75 }\end{array}$ & 1.178 & CCXLI & Approx. 13c \\
\hline $\begin{array}{l}\text { Add. 17,224, } \\
\text { fol. 76 }\end{array}$ & 3.1198 & MXXVI & Approx. 11c-12c \\
\hline $\begin{array}{l}\text { Add. 17,224, } \\
\text { fol. 77 }\end{array}$ & 3.1199 & MXXX & Approx. 13c \\
\hline $\begin{array}{l}\text { Add. 17,224, } \\
\text { foll. 1-17 }\end{array}$ & 1.306 & CCCLXXVI & Approx. 13c \\
\hline $\begin{array}{l}\text { Add. 17,224, } \\
\text { foll. 19-21 }\end{array}$ & $1.174-175$ & CCXXXIII & Approx. 13c-14c \\
\hline $\begin{array}{l}\text { Add. 17,224, } \\
\text { foll. 22-33 }\end{array}$ & $1.173-174$ & CCXXX & Approx. 13c \\
\hline $\begin{array}{l}\text { Add. 17,224, } \\
\text { foll. 34, 35 }\end{array}$ & 1.194 & CCXLIX & Approx. 12c-13c \\
\hline $\begin{array}{l}\text { Add. 17,224, } \\
\text { foll. 37-42 }\end{array}$ & $1.69-70$ & CIX & Approx. 13c \\
\hline $\begin{array}{l}\text { Add. 17,224, } \\
\text { foll. 43-57 }\end{array}$ & 1.67 & XCVIII & 1173 \\
\hline $\begin{array}{l}\text { Add. 17,224, } \\
\text { foll. 58-65 }\end{array}$ & 1.66 & CXV \\
\hline $\begin{array}{l}\text { Add. 17,224, } \\
\text { foll. 67-70 }\end{array}$ & 1.95 & CXLIX & Approx. 13c \\
\hline $\begin{array}{l}\text { Add. 17,224, } \\
\text { foll. 71, 72 }\end{array}$ & 1.95 & CLI \\
\hline $\begin{array}{l}\text { Add. 17,224, } \\
\text { foll. 73, 74 }\end{array}$ & 1.95 & Approx. 13c \\
\hline Add. 17,225 & $1.72-73 \mathrm{c}$ \\
\hline Add. 17,226 & 1.79 & Approx. 13c-14c \\
\hline
\end{tabular}




\begin{tabular}{|c|c|c|c|}
\hline Shelfmark & $\begin{array}{l}\text { Catalog pp. } \\
\text { (volume. } \\
\text { pages) }\end{array}$ & $\begin{array}{l}\text { Wright's } \\
\text { Numeration }\end{array}$ & Wright's Dating \\
\hline Add. 17,227 & 1.94-95 & CXLVII & 1254 \\
\hline $\begin{array}{l}\text { Add. 17,228, } \\
\text { foll. 1-37 }\end{array}$ & 1.96 & CLIII & Approx. 13c \\
\hline $\begin{array}{l}\text { Add. 17,228, } \\
\text { foll. } 38-64\end{array}$ & 1.83 & CXXXI & Approx. 13c \\
\hline $\begin{array}{l}\text { Add. 17,229, } \\
\text { foll. 1-47 }\end{array}$ & $1.207-208$ & CCLXIII & 1218 \\
\hline $\begin{array}{l}\text { Add. 17,229, } \\
\text { foll. 48-77 }\end{array}$ & 1.209 & CCLXV & Approx. 13c \\
\hline $\begin{array}{l}\text { Add. 17,230, } \\
\text { foll. 1-19 }\end{array}$ & 1.234 & CCCI & Approx. 14c \\
\hline $\begin{array}{l}\text { Add. 17,230, } \\
\text { foll. } 20-46\end{array}$ & $1.234-235$ & CCCII & 1337 \\
\hline Add. 17,231 & $1.299-301$ & CCCLXIV & 1484 \\
\hline Add. 17,232 & $1.371-374$ & CCCCLXIX & 1210 \\
\hline $\begin{array}{l}\text { Add. 17,233, } \\
\text { foll. 1-79 }\end{array}$ & $1.316-317$ & CCCCVI & Approx. 13c \\
\hline $\begin{array}{l}\text { Add. 17,233, } \\
\text { foll. } 80-151\end{array}$ & $1.325-326$ & CCCCXVI & Approx. 13c \\
\hline Add. 17,234 & $1.317-318$ & CCCCVII & Approx. 13c \\
\hline Add. 17,235 & $1.276-277$ & CCCXXXIII & $\begin{array}{l}\text { Approx. } \\
\text { (early) }\end{array}$ \\
\hline Add. 17,236 & $1.318-320$ & CCCCVIII & 1284 \\
\hline Add. 17,237 & $1.306-307$ & CCCLXXVIII & Approx. $13 \mathrm{c}-14 \mathrm{c}$ \\
\hline Add. 17,238 & $1.353-354$ & CCCCXXXIX & Approx. 13c \\
\hline $\begin{array}{l}\text { Add. 17,239, } \\
\text { foll. 1-15 }\end{array}$ & $1.212-213$ & CCLXXVIII & Approx. 14c \\
\hline $\begin{array}{l}\text { Add. 17,239, } \\
\text { foll. 16-60 }\end{array}$ & 1.314 & CCCXCVI & 1518 \\
\hline Add. 17,240 & $1.326-327$ & CCCCXVII & Approx. 13c \\
\hline Add. 17,241 & 1.312 & CCCXCII & Approx. 13c \\
\hline
\end{tabular}




\begin{tabular}{|l|l|l|l|}
\hline Shelfmark & $\begin{array}{l}\text { Catalog pp. } \\
\text { (volume. } \\
\text { pages) }\end{array}$ & $\begin{array}{l}\text { Wright's } \\
\text { Numeration }\end{array}$ & Wright's Dating \\
\hline $\begin{array}{l}\text { Add. 17,242, } \\
\text { foll. 1-104 }\end{array}$ & 2.504 & DCXXXV & Approx. 11c-12c \\
\hline $\begin{array}{l}\text { Add. 17,242, } \\
\text { foll. 105-127 }\end{array}$ & 2.517 & DCLXVII & Approx. 12c \\
\hline Add. 17,243 & $1.290-291$ & CCCXLV & Approx. 13c \\
\hline Add. 17,244 & 1.354 & CCCCXL & Approx. 13c \\
\hline Add. 17,245 & $1.301-302$ & CCCLXVI & Approx. 13c \\
\hline $\begin{array}{l}\text { Add. 17,246, } \\
\text { foll. 1-74 }\end{array}$ & $1.307-308$ & CCCLXXX & 1239 \\
\hline $\begin{array}{l}\text { Add. 17,246, } \\
\text { foll. 75-84 }\end{array}$ & 1.308 & CCCLXXXI & 1239 \\
\hline $\begin{array}{l}\text { Add. 17,246, } \\
\text { foll. 85-90 }\end{array}$ & 1.308 & CCCLXXXII & Approx. 14c \\
\hline Add. 17,247 & $1.350-351$ & CCCCXXXVI & Approx. 12c \\
\hline Add. 17,248 & 1.369 & CCCCLXIV & Approx. 12c \\
\hline Add. 17,249 & $1.303-304$ & CCCLXXIII & Approx. 13c-14c \\
\hline Add. 17,250 & $1.313-314$ & CCCXCV & Approx. 13c \\
\hline Add. 17,251 & $1.354-355$ & CCCCXLI & Approx. 13c \\
\hline Add. 17,252 & $1.296-297$ & CCCLVIII & Approx. 13c-14c \\
\hline $\begin{array}{l}\text { Add. 17,253, } \\
\text { foll. 1-69 }\end{array}$ & $1.352-353$ & CCCCXXXVIII & 1222 \\
\hline $\begin{array}{l}\text { Add. 17,253, } \\
\text { foll. 70-103 }\end{array}$ & 1.381 & CCCCLXXXIX & Approx. 13c \\
\hline Add. 17,254 & $1.355-356$ & CCCCXLII & Approx. 13c \\
\hline Add. 17,255 & $1.357-358$ & CCCCXLVII & Approx. 13c \\
\hline $\begin{array}{l}\text { Add. 17,256, } \\
\text { foll. 1-82 }\end{array}$ & $1.141-142$ & CCVIII & 1251 \\
\hline $\begin{array}{l}\text { Add. 17,256, } \\
\text { foll. 83-132 }\end{array}$ & $1.142-143$ & CCIX & 1251 \\
\hline Add. 17,257, & 1.138 & CXCIX & Approx. 14c \\
\hline
\end{tabular}




\begin{tabular}{|c|c|c|c|}
\hline Shelfmark & $\begin{array}{l}\text { Catalog pp. } \\
\text { (volume. } \\
\text { pages) }\end{array}$ & $\begin{array}{l}\text { Wright's } \\
\text { Numeration }\end{array}$ & Wright's Dating \\
\hline \multicolumn{4}{|l|}{ fol. 112} \\
\hline $\begin{array}{l}\text { Add. } 17,257 \text {, } \\
\text { fol. } 113\end{array}$ & 1.138 & $\mathrm{CC}$ & Approx. 14c-15c \\
\hline $\begin{array}{l}\text { Add. 17,257, } \\
\text { fol. } 74\end{array}$ & 1.141 & CCVI & Approx. 12c-13c \\
\hline $\begin{array}{l}\text { Add. 17,257, } \\
\text { fol. } 75\end{array}$ & 1.143 & CCXIII & Approx. 13c \\
\hline $\begin{array}{l}\text { Add. 17,257, } \\
\text { fol. } 79\end{array}$ & $1.136-137$ & CXCIV & Approx. 13c-14c \\
\hline $\begin{array}{l}\text { Add. 17,257, } \\
\text { fol. } 80\end{array}$ & 1.143 & CCXII & Approx. 13c \\
\hline $\begin{array}{l}\text { Add. 17,257, } \\
\text { fol. } 81\end{array}$ & 1.144 & CCXVI & Approx. 14c \\
\hline $\begin{array}{l}\text { Add. 17,257, } \\
\text { foll. 1-21 }\end{array}$ & 1.141 & CCVII & 1248 \\
\hline $\begin{array}{l}\text { Add. 17,257, } \\
\text { foll. 103-107 }\end{array}$ & 1.137 & $\mathrm{CXCV}$ & Approx. 13c-14c \\
\hline $\begin{array}{l}\text { Add. 17,257, } \\
\text { foll. 108-111 }\end{array}$ & 1.137 & CXCVI & Approx. 13c-14c \\
\hline $\begin{array}{l}\text { Add. 17,257, } \\
\text { foll. } 22-73\end{array}$ & $1.143-144$ & CCXIV & Approx. 13c-14c \\
\hline $\begin{array}{l}\text { Add. 17,257, } \\
\text { foll. } 76-78\end{array}$ & 1.144 & CCXV & Approx. 13c-14c \\
\hline $\begin{array}{l}\text { Add. 17,257, } \\
\text { foll. } 82,83\end{array}$ & 1.129 & CLXXXV & Approx. 12c \\
\hline $\begin{array}{l}\text { Add. 17,257, } \\
\text { foll. 84-94 }\end{array}$ & 1.37 & LVI & Approx. 13c \\
\hline $\begin{array}{l}\text { Add. 17,257, } \\
\text { foll. 95-102 }\end{array}$ & $1.138-139$ & CCI & Approx. 15c \\
\hline $\begin{array}{l}\text { Add. 17,258, } \\
\text { foll. 1-118 }\end{array}$ & 1.381 & CCCCXCI & Approx. 13c-14c \\
\hline $\begin{array}{l}\text { Add. 17,258, } \\
\text { foll. 119-190 }\end{array}$ & 1.328 & CCCCXIX & Approx. 13c \\
\hline
\end{tabular}




\begin{tabular}{|l|l|l|l|}
\hline Shelfmark & $\begin{array}{l}\text { Catalog pp. } \\
\text { (volume. } \\
\text { pages) }\end{array}$ & $\begin{array}{l}\text { Wright's } \\
\text { Numeration }\end{array}$ & Wright's Dating \\
\hline Add. 17,259 & $1.356-357$ & CCCCXLIII & Approx. 13c \\
\hline Add. 17,260 & $1.397-398$ & DXXI & Approx. 12c-13c \\
\hline $\begin{array}{l}\text { Add. 17,261, } \\
\text { foll. 1-8 }\end{array}$ & 1.357 & CCCCXLV & Approx. 13c \\
\hline $\begin{array}{l}\text { Add. 17,261, } \\
\text { foll. 67-149 }\end{array}$ & 1.314 & CCCXCVII & Approx. 16c \\
\hline $\begin{array}{l}\text { Add. 17,261, } \\
\text { foll. 9-66 }\end{array}$ & $1.358-359$ & CCCCXLIX & Approx. 13c-14c \\
\hline Add. 17,262 & $2.867-873$ & DCCCXXXVII & Approx. 12c \\
\hline Add. 17,263 & $3.1079-1080$ & DCCCCXXI & Approx. 13c \\
\hline Add. 17,264 & $3.1078-1079$ & DCCCCXX & Approx. 13c \\
\hline Add. 17,265 & $3.1140-1141$ & DCCCCLXII & Approx. 13c \\
\hline $\begin{array}{l}\text { Add. 17,266, } \\
\text { foll. 1-50 }\end{array}$ & 2.865 & DCCCXXXIII & $\begin{array}{l}\text { Approx. } \\
\text { (early) }\end{array}$ \\
\hline $\begin{array}{l}\text { Add. 17,266, } \\
\text { foll. 51-78 }\end{array}$ & $1.128-129$ & CLXXXIII & Approx. 12c \\
\hline $\begin{array}{l}\text { Add. 17,267, } \\
\text { foll. 1-8 }\end{array}$ & 2.481 & DCII & Approx. 13c \\
\hline $\begin{array}{l}\text { Add. 17,267, } \\
\text { foll. 13-22 }\end{array}$ & $2.884-885$ & DCCCXLV & Approx. 13c \\
\hline $\begin{array}{l}\text { Add. 17,267, } \\
\text { foll. 23-33 }\end{array}$ & 2.885 & DCCCXLVI & Approx. 13c \\
\hline $\begin{array}{l}\text { Add. 17,267, } \\
\text { foll. 34-49 }\end{array}$ & $2.885-886$ & DCCCXLVII & Approx. 13c \\
\hline $\begin{array}{l}\text { Add. 17,267, } \\
\text { foll. 50-75 }\end{array}$ & 3.1146 & DCCCCLXIV & Approx. 13c \\
\hline $\begin{array}{l}\text { Add. 17,267, } \\
\text { foll. 9-12 }\end{array}$ & 2.884 & DCCCXLIV & Approx. 13c \\
\hline Add. 17,268 & 1.129 & CLXXXIV & Approx. 12c \\
\hline $\begin{array}{l}\text { Add. 17,269, } \\
\text { foll. 1-38 }\end{array}$ & 1.382 & CCCCXCIII & 1499 \\
\hline
\end{tabular}




\begin{tabular}{|c|c|c|c|}
\hline Shelfmark & $\begin{array}{l}\text { Catalog pp. } \\
\text { (volume. } \\
\text { pages) }\end{array}$ & $\begin{array}{l}\text { Wright's } \\
\text { Numeration }\end{array}$ & Wright's Dating \\
\hline $\begin{array}{l}\text { Add. } 17,269 \text {, } \\
\text { foll. } 39-80\end{array}$ & 1.357 & CCCCXLIV & Approx. 13c \\
\hline $\begin{array}{l}\text { Add. 17,269, } \\
\text { foll. } 81-88\end{array}$ & 1.213 & CCLXXXI & Approx. 15c \\
\hline $\begin{array}{l}\text { Add. 17,269, } \\
\text { foll. } 89-92\end{array}$ & 1.378 & CCCCLXXXI & Approx. 14c \\
\hline Add. 17,270 & 2.482 & $\mathrm{DCV}$ & Approx. 9c \\
\hline Add. 17,271 & $1.297-298$ & CCCLX & Approx. $12 c-13 c$ \\
\hline $\begin{array}{l}\text { Add. 17,272, } \\
\text { foll. 1-29 }\end{array}$ & 1.311 & CCCLXXXVIII & Approx. 13c \\
\hline $\begin{array}{l}\text { Add. 17,272, } \\
\text { foll. 100-105 }\end{array}$ & 1.296 & CCCLVI & Approx. 13c \\
\hline $\begin{array}{l}\text { Add. 17,272, } \\
\text { foll. 106-112 }\end{array}$ & 1.324 & CCCCXI & Approx. 13c \\
\hline $\begin{array}{l}\text { Add. 17,272, } \\
\text { foll. 30-63 }\end{array}$ & 2.517 & DCLXXI & 1565 \\
\hline $\begin{array}{l}\text { Add. 17,272, } \\
\text { foll. } 64-67\end{array}$ & 3.1148 & DCCCCLXVIII & Approx. 12c \\
\hline $\begin{array}{l}\text { Add. } 17,272 \text {, } \\
\text { foll. } 68-74\end{array}$ & $2.1022-1023$ & DCCCLXXIX & Approx. 13c \\
\hline $\begin{array}{l}\text { Add. 17,272, } \\
\text { foll. 75-99 }\end{array}$ & 1.298 & CCCLXI & Approx. 13c \\
\hline Add. 17,273 & $1.347-349$ & CCCCXXXI & Approx. 9c \\
\hline Add. 17,274 & 2.620 & DCCXX & Approx. 11c-12c \\
\hline Add. 17,922 & 1.60 & LXXXIV & $1222-3$ \\
\hline Add. 17,923 & $1.182-188$ & CCXLVI & $11 \mathrm{c}$ \\
\hline Add. 17,983 & $1.61-62$ & LXXXVI & 1438 \\
\hline Add. 18,295 & 3.1183-1184 & MII & 1603 \\
\hline Add. 18,296 & $2.628-630$ & DCCXXV & 1714 \\
\hline Add. 18,714 & $1.161-167$ & CCXXVI & 1214 \\
\hline
\end{tabular}




\begin{tabular}{|l|l|l|l|}
\hline Shelfmark & $\begin{array}{l}\text { Catalog pp. } \\
\text { (volume. } \\
\text { pages) }\end{array}$ & $\begin{array}{l}\text { Wright's } \\
\text { Numeration }\end{array}$ & Wright's Dating \\
\hline Add. 18,715 & $1.18-20$ & XXXII & Approx. 12c \\
\hline Add. 18,716 & $2.901-903$ & DCCCLI & Approx. 15c-16c \\
\hline Add. 18,812 & 1.83 & CXXIX & Approx. 6c-7c \\
\hline Add. 18,813 & $2.729-731$ & DCCLXIII & Approx. 7c \\
\hline $\begin{array}{l}\text { Add. 18,814, } \\
\text { foll. 1-102 }\end{array}$ & $2.734-736$ & DCCLXVII & Approx. 7c-8c \\
\hline $\begin{array}{l}\text { Add. 18,814, } \\
\text { foll. 103-262 }\end{array}$ & $2.793-796$ & DCCXCVII & Approx. 9c \\
\hline Add. 18,815 & $2.436-437$ & DLIX & Approx. 9c \\
\hline Add. 18,816 & $1.339-340$ & CCCCXXII & Approx. 9c \\
\hline Add. 18,817 & $2.803-806$ & DCCCI & Approx. 9c \\
\hline Add. 18,818 & 2.489 & DCXVII & Approx. 7c \\
\hline $\begin{array}{l}\text { Add. 18,819, } \\
\text { foll. 1-91 }\end{array}$ & $1.340-341$ & CCCCXXIV & Approx. 9c-10c \\
\hline $\begin{array}{l}\text { Add. 18,819, } \\
\text { foll. 110-120 }\end{array}$ & 1.345 & CCCCXXVIII & Approx. 10c-11c \\
\hline $\begin{array}{l}\text { Add. 18,819, } \\
\text { foll. 92-109 }\end{array}$ & 1.340 & CCCCXXIII & 884 \\
\hline $\begin{array}{l}\text { Add. 18,820, } \\
\text { foll. 1-16 }\end{array}$ & 1.276 & CCCXXXII & Approx. 12c \\
\hline $\begin{array}{l}\text { Add. 18,820, } \\
\text { foll. 17-55 }\end{array}$ & $1.269-270$ & CCCXXIII & Approx. 11c-12c \\
\hline Add. 18,821 & $2.775-776$ & DCCLXXXVI & Approx. 9c \\
\hline Add. 21,031 & $1.327-328$ & CCCCXVIII & 1213 \\
\hline Add. 21,210 & $2.876-882$ & DCCCXLI & 1242 \\
\hline Add. 21,211 & $3.1180-1182$ & MI & 1831 \\
\hline Add. 21,454 & $3.1165-1167$ & DCCCCXCV & Approx. 13c \\
\hline Add. 21,580 & $2.624-626$ & DCCXXIII & 1478 \\
\hline Add. 22,370 & $2.500-501$ & DCXXIX & Approx. 14c-15c \\
\hline Add. 23,596 & $2.626-628$ & DCCXXIV & 1720 \\
\hline
\end{tabular}




\begin{tabular}{|l|l|l|l|}
\hline Shelfmark & $\begin{array}{l}\text { Catalog pp. } \\
\text { (volume. } \\
\text { pages) }\end{array}$ & $\begin{array}{l}\text { Wright's } \\
\text { Numeration }\end{array}$ & Wright's Dating \\
\hline Add. 23,597 & $3.1173-1174$ & DCCCCXCVIII & Approx. 18c \\
\hline Add. 25,874 & $1.213-214$ & CCLXXXII & 1740 \\
\hline Add. 25,875 & $3.1064-1069$ & DCCCCXXII & $1709-10$ \\
\hline Add. 25,876 & $3.1174-1179$ & DCCCCXCIX & Approx. 16c \\
\hline Add. 25,877 & 3.1179 & M & 1733 \\
\hline $\begin{array}{l}\text { Add. 25,878, } \\
\text { foll. 1-70 }\end{array}$ & $3.1192-1193$ & MVIII & Approx. 17c \\
\hline $\begin{array}{l}\text { Add. 25,878, } \\
\text { foll. 71-87 }\end{array}$ & 1.139 & CCII & Approx. 15c \\
\hline Add. 26,552 & $1.137-138$ & CXCVIII & Approx. 14c \\
\hline Egerton 681 & $1.190-194$ & CCXLVIII & $1206-07$ \\
\hline Egerton 703 & $1.237-239$ & CCCV & 1683 \\
\hline Egerton 704 & $1.1-3$ & I & Approx. 17c \\
\hline Egerton 709 & $3.1190-1191$ & MVII & Approx. 16c \\
\hline Harl. 5512 & $1.214-216$ & CCLXXXIII & 1549 \\
\hline Orient. 1017 & $2.890-901$ & DCCCL & 1364 \\
\hline Sloane 3597 & $1.236-237$ & CCCIV & $1701-2$ \\
\hline
\end{tabular}

